\title{
15. A Sr-, Nd-, Pb-, O-ISOTOPE STUDY OF PLUTONIC ROCKS FROM MARK, LEG 153: IMPLICATIONS FOR MANTLE HETEROGENEITY AND MAGMA CHAMBER PROCESSES ${ }^{1}$
}

\author{
Pamela D. Kempton ${ }^{2}$ and Arlëne G. Hunter ${ }^{2}$
}

\begin{abstract}
Drilling on the western wall of the Mid-Atlantic Ridge south of the Kane Fracture Zone at MARK $\left(23^{\circ} 20^{\prime} \mathrm{N}\right.$ and $\left.23^{\circ} 30^{\prime} \mathrm{N}\right)$ recovered plutonic rocks < $1 \mathrm{Ma}$ in age. A selection of these rocks has been analyzed for their $\mathrm{Sr}-, \mathrm{Nd}-, \mathrm{Pb}-$, and $\mathrm{O}$-isotope compositions. O-isotope analyses of gabbro mineral separates have near primary magmatic values. $\delta^{18} \mathrm{O}_{\mathrm{cpx}}$ vs. $\delta^{18} \mathrm{O}_{\mathrm{plag}}$ systematics support only limited fluid-rock interaction, predominantly at moderate to low temperatures $\left(200^{\circ}-300^{\circ} \mathrm{C}\right)$. Combined $\mathrm{Sr}$ - and $\mathrm{O}$-isotope data indicate that, even where fluids have found ingress into the gabbroic crust, they have had limited compositional consequences, presumably because seawater was rapidly modified by interaction with the overlying basalt crust. $\mathrm{Pb}$ - and $\mathrm{Sr}-$ isotope compositions of leachates further demonstrate that, for most samples, seawater was the principal component in hydrothermal fluids; in a few cases, however, sediments were also involved.

$\mathrm{Sr}-, \mathrm{Nd}-$, and $\mathrm{Pb}$-isotope systematics of leached gabbro mineral separates indicate that, to a first-order approximation, the sub-MARK mantle is isotopically homogeneous from near the middle of the spreading cell (Sites 921-924) to its boundary in the south (Site 920). Trace-element concentrations of $\mathrm{Sr}, \mathrm{Nd}$, and $\mathrm{Pb}$ (and, by implication, other incompatible trace elements) must also be homogeneous. Thus, variations in trace-element concentrations in gabbros and calculated parental magmas must be the product of the melting regime and subsequent fractionation processes in the magma chamber(s) and not variations in the mantle source composition.

Superimposed on this homogeneity is smaller scale, within-sample, isotope disequilibrium between coexisting plagioclase and clinopyroxene. Such heterogeneities provide evidence of complex magma chamber processes and are most likely to be preserved at the beginning of magmatic cycles. Unusual isotopically enriched compositions are found in the MARK area as diabase and amphibolitized microgabbros that crosscut the peridotite section. Restriction of these distinctive compositions in the peridotite sections at Site 920 may result from the lower magma supply rate expected near the boundary of a spreading cell.

The average isotopic composition within the MARK area has changed over a period of $<1 \mathrm{~m}$.y., becoming more radiogenic in its $\mathrm{Pb}$-isotope composition but less radiogenic in its $\mathrm{Nd}$ composition, implying that the source is currently less depleted than that available $750,000 \mathrm{yr}$ earlier.
\end{abstract}

\section{INTRODUCTION}

There is a growing consensus that the range of major-element, trace-element, and isotopic compositions of magmas supplied to the mid-ocean ridge system is far larger than that observed in the erupted products. This was first suspected from studies of melt inclusions trapped in phenocrysts that were in many cases more primitive than any erupted magma composition (Dungan and Rhodes, 1978; Humler and Whitechurch, 1988; Sobolev and Shimizu, 1993). Similarly, although basalts dredged from the Southwest Indian Ridge (Johnson and Dick, 1992; Snow, 1993) overlap the isotopic compositions of gabbros from Hole 735B (Kempton et al., 1991), the gabbros extend to higher ${ }^{143} \mathrm{Nd} /{ }^{144} \mathrm{Nd}$ and to lower ${ }^{206} \mathrm{~Pb} /{ }^{204} \mathrm{~Pb}$ and ${ }^{208} \mathrm{~Pb} /{ }^{204} \mathrm{~Pb}$ ratios. In addition, recent studies of abyssal peridotites (Johnson et al., 1990; Johnson and Dick, 1992) have provided clear evidence that melting in the suboceanic mantle is in many cases polybaric, beginning in the garnet stability field. One important implication of these studies is that a wide range of melt compositions can be generated as a function of changing mineralogy, pressure, and temperature during mantle melting beneath mid-ocean ridges. However, in contrast to theory, the compositional spectrum observed within mid-ocean ridge basalts is relatively small. It has been argued that this is because the various end members become homogenized before eruption, either during ascent in the mantle, or in crustal magma chambers, or both (Klein and Langmuir, 1987; Johnson et al., 1990). If the model is correct, cumu-

'Karson, J.A., Cannat, M., Miller, D.J., and Elthon, D. (Eds.), 1997. Proc. ODP, Sci. Results, 153: College Station, TX (Ocean Drilling Program).

${ }^{2}$ NERC Isotope Geosciences Laboratory, Kingsley Durham Centre, Keyworth, NG12 5GG, United Kingdom. P.KEMPTON@NIGL.NERC.AC.UK late gabbroic rocks might be expected to record much more variability than the spatially associated basalts. This is because a greater proportion of the spectrum of melt compositions entering a magma chamber can be preserved as distinct cumulus phases or layers, as long as they crystallize before significant magma homogenization. In addition, the cumulates may also have been influenced by circulating intercumulus liquids during solidification, both in the absence of deformation and during synkinematic differentiation (Dick et al., 1991).

Unfortunately, the amount of published trace-element and isotopic data for plutonic oceanic rocks is very small, particularly for $\mathrm{Pb}$ isotopes. Thus, simply documenting the isotopic characteristics of oceanic cumulates and residual mantle peridotites on the level of their constituent minerals is a necessary prelude to addressing more fundamental questions of mantle heterogeneity, magma chamber processes, and ocean spreading dynamics.

This paper presents new $\mathrm{Sr}-, \mathrm{Nd}-$ and $\mathrm{Pb}$-isotope data on a selection of gabbros drilled during Ocean Drilling Program (ODP) Leg 153 to the Mid-Atlantic Ridge, south of the Kane Fracture Zone (MARK). Also included are new isotope data on fresh basalt glasses dredged from the neovolcanic zone. These data will be used in conjunction with data on peridotites (Kempton and Stephens, this volume) and diabase dikes (Kempton and Casey, this volume) also recovered during Leg 153 to address the problem of heterogeneity within the mantle beneath the MARK area. In particular we will (1) assess the horizontal scale of isotopic heterogeneity by comparing drill sites and drill holes; (2) assess the isotopic heterogeneity within a hole, and by implication the variation in source composition over the life time of the magma chamber(s) represented by the stratigraphic column; (3) determine whether crosscutting gabbro dikes from the peridotite massif at Site 920 are genetically related to the massif gab- 
bros from Sites 921 to 924 ; (4) determine whether the MARK peridotites represent the source composition of the gabbros and/or erupted basalts; and (5) determine whether the basalts presently erupted within the neovolcanic zone originated from the same source as that which gave rise to the gabbros.

\section{BACKGROUND}

The MARK area is characterized by slow-spreading, probable low degrees of mantle melting and magma supply, and basalt compositions that are typical of N-MORB (non-plume). Coarse-grained mafic and ultramafic rocks, thought to be approximately $0.75 \mathrm{Ma}$, are exposed along the median valley walls. The rocks are thought to have been exposed as a result of mechanical extension of the axial lithosphere during periods of limited crustal formation. The area includes two distinct ridge segments that have contrasting deep crustal structures. The northern cell, which extends from the nodal basin down to $23^{\circ} 18^{\prime} \mathrm{N}$, has a seismic crustal thickness of $4-5 \mathrm{~km}$; the Snake Pit hydrothermal field is located in this segment. In constrast, the southern cell has a seismic crustal thickness of 6-7 km (Purdy and Detrick, 1986). A broad transition zone of significantly thinner crust separates the two domains and has been interpreted as a zero-offset transform fault (Purdy and Detrick, 1986) or an "accommodation zone" (Karson, 1991). Fifteen holes were drilled at five sites (Sites 920-924), four in peridotite, and eleven in gabbro. A summary of the holes drilled and basic recovery information is listed in Figure 1. The gabbro sites (Sites 921-924) are located approximately in the middle of the northern domain, whereas the peridotite site (Site 920) is located near the offset of the northern and southern spreading cells at $23^{\circ} 21^{\prime} \mathrm{N}$ in an area of presumed thin crust.

\section{Site 920: Mantle Peridotites}

Ultramafic rocks were recovered only at Site 920. The four holes drilled at Site 920 met with differing degrees of success, but nearly $150 \mathrm{~m}$ of peridotite was recovered from a total of $340 \mathrm{~m}$ drilled at Holes 920B and 920D (42\% cumulative recovery). These two holes are only $20 \mathrm{~m}$ apart and several units appear to be traceable between them (Shipboard Scientific Party, 1995a). Nevertheless, mineralogical differences between the cores document the significant heterogeneity even over this short lateral distance. The predominant lithology recovered was coarse-grained porphyroclastic harzburgite, now about $80 \%$ altered to serpentine (plus lesser amounts of tremolite-actinolite, chlorite, magnetite, and carbonate minerals). Serpentinized lherzolite, dunite, and websterite were less commonly encountered. On average, the peridotite section is very depleted, with an estimated mode (pre-serpentinization) of $81 \%$ olivine, $16 \%$ orthopyroxene, $2 \%$ clinopyroxene, and $1 \%$ spinel; by comparison, average abyssal peridotite has $75 \%$ olivine, $21 \%$ orthopyroxene, $4 \%$ clinopyroxene, and $<1 \%$ spinel (Dick et al., 1994).

The ultramafic assemblages are crosscut by layers or veins of clinopyroxenite, gabbro, olivine gabbro, oxide-rich metagabbro, oxiderich gabbronorite, oxide-rich clinopyroxenite, amphibolitized microgabbro, hornblendite, and rodingitized gabbro. The peridotites are also crosscut by unusual $\mathrm{Mg}$-rich (13-15 wt $\% \mathrm{MgO}$ ) plagioclaseolivine-phyric diabase dikes (Kempton and Casey, this volume).

\section{Sites 921-924: Gabbroic Rocks}

Nearly $120 \mathrm{~m}$ of gabbroic rock was recovered from a total of 447 $\mathrm{m}$ drilled at Sites 921, 922, 923, and 924 (27\% cumulative recovery). Again, the various holes drilled met with different degrees of success, but Holes $921 \mathrm{E}$ and $923 \mathrm{~A}$ stand out in terms of material recovered. The gabbros are characterized by a wide range in composition, grain size, texture, extent of alteration, and deformation. The predominant

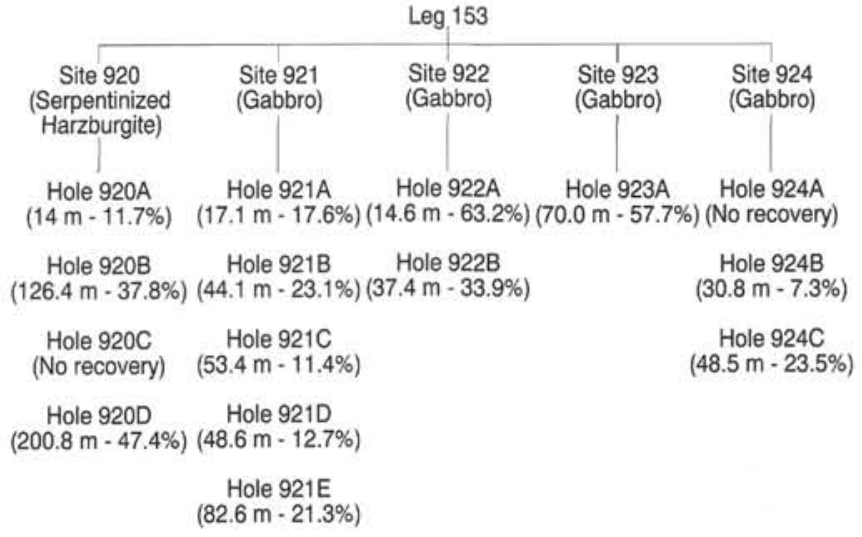

Figure 1. Summary of Leg 153 drilling results. Numbers in parentheses indicate meters of rock recovered and percent recovery.

lithologies recovered are gabbro, olivine gabbro, and troctolite, but volumetrically minor oxide gabbro, oxide gabbronorite, gabbronorite, trondhjemite, dacite, and diabase also occur. Many gabbroic rocks retain igneous textures, but a wide range of crystal-plastic to cataclastic deformation fabrics is also developed, particularly near the top of the holes. Alteration is generally very low $(<10 \%)$, except in areas of intense deformation. Primary magmatic layering occurs as changes in grain size and/or modal mineralogy with variations on a scale of $1 \mathrm{~cm}$ up to a few meters.

Relatively high recovery in Hole 923A provided significant insights into layering patterns that are only partially preserved in the other gabbro holes. In this hole, preliminary mesoscopic and thinsection observations suggest the occurrence of cycles of relatively primitive to more evolved rocks that may indicate recurrent evolutionary magmatic sequences (Shipboard Scientific Party, 1995b; Stephens et al., this volume). Compositional cycles consistent with an evolutionary magmatic sequence were tentatively identified within the gabbroic stratigraphy, based on variations in modal mineralogy, texture, and inferred changes in mineral composition observed during macroscopic description of the core. These cycles record, to varying degrees, a transition from a primitive olivine gabbro or troctolite at the base, upward through olivine gabbro in which green clinopyroxene occurs as an oikocryst phase, into olivine gabbro and gabbro in which brown pyroxene is a cumulus phase. Such magmatic cyclicity was not observed in gabbros drilled during Leg 118 (Hole 735B) or Leg 147 to Hess Deep.

\section{COMPARISON WITH PLUTONIC ROCKS FROM LEGS 118 AND 147}

The MARK peridotite sections differ subtly from those recovered during Leg 147, particularly with respect to the smaller amount of dunite observed at MARK (Dick et al., 1994). However, both localities provide evidence for a close relationship between depletion in the peridotites and the presence of mafic veins or dikes. Dick et al. (1994) interpreted the dunites at Hess Deep as evidence for melt-rock reactions. Whether these depleted zones are the cause or the effect of melt migration is the subject of several contributions to this volume (e.g., Niida, this volume; Cannat, Chatin, et al., this volume; Casey, this volume).

Hess Deep and MARK also differ in the types of gabbroic rocks recovered in that primitive troctolites were not found at Hess Deep but are very common at MARK. There are remarkable similarities in the MARK and Hole 735B gabbroic sections, but the relative proportions of rock types in the two localities differ significantly. In particular, in common with Hess Deep, oxide-rich ferrogabbros are rela- 
tively minor at MARK, but these lithologies are extremely abundant at Hole 735B. Furthermore, magmatic cyclicity was apparently not observed in gabbros from Hole 735B nor from Leg 147.

There are also similarities between Hole $735 \mathrm{~B}$ and MARK in structural style. Detailed study of the Hole 735B samples indicates that magmatic and deformational processes are strongly intermingled (Dick et al., 1994); deformation started before the rocks were completely cooled, creating a planar magmatic foliation. Formation of shear zones facilitated early seawater penetration in the deep crust and consequent reaction with rocks at high temperature. Preliminary interpretations of the structures and stratigraphy encountered during Leg 153 suggest that similar features may exist at MARK, but that compositional effects associated with influx of seawater are limited (Agar et al., this volume). Rocks encountered at the tops of most holes in the gabbro massif are typically more deformed and altered than those lower down, suggesting that the exposed surfaces are major fault planes. However, most of the holes drilled during Leg 153 were relatively shallow compared with Hole $735 \mathrm{~B}$, and it is unclear whether further deformation would have been encountered if drilling had continued to greater depths.

\section{SAMPLE SELECTION AND PETROGRAPHY}

Because of the survey nature of this preliminary study, samples were selected to encompass as much of the lithologic, stratigraphic, and geographic variation as possible. Included are massif gabbros from Sites 921 to 924 , as well as gabbroic veins/dikelets crosscutting the peridotite section of Site 920 . In particular, we analyzed seven olivine gabbros (Holes 921B, 922B, 923A, and 924B), two oxide gabbros (Hole 920D and 922A), one pegmatitic brown gabbro (Hole 921E), one gneissic oxide-bearing gabbro (Hole 920B), and one amphibolitized microgabbro (Hole 920B). Petrographic descriptions of, and isotope data for, peridotites from Site 920 are presented in a separate report (Kempton and Stephens, this volume), as are data for crosscutting diabase dikes from Sites 921 and crosscutting Mg-rich dikes from Site 920 (Kempton and Casey, this volume). In addition, recent basalts dredged from the central neovolcanic zone (Reynolds, 1995) are included in this study for comparison with the older plutonic and hypabyssal rocks. Detailed descriptions of individual samples analyzed in this study are provided in the appendix, but a general summary of lithologic characteristics and rationale for sample selection are provided here.

The gabbros recovered during Leg 153 exhibit a wide range of compositions and textures, but useful distinctions can be made on the basis of mineralogical characteristics. The most primitive (high $\mathrm{Mg}$, $\mathrm{Cr}$, etc.) gabbros are olivine rich, ranging from olivine gabbro to troctolite. They are distinguished by the occurrence of oikocrystic clinopyroxene and olivine that poikilitically enclose euhedral to subhedral plagioclase laths. Classic crescumulate textures have been observed in some samples (e.g., Sample 153-923A-12R-1, 30-38 cm). Clinopyroxene occurs both as a cumulus and as an intercumulus phase, but this group is typified by bright green oikocrysts that may be several centimeters in size. In some cases, the clinopyroxene is zoned from green to brown toward the crystal rims. Iron oxide minerals are rare. Mineral proportions and textures are extremely variable, and primary magmatic layering is usually restricted to diffuse modal variations. Samples of this type analyzed for this study include the following: Sample 153-921B-4R-2, 76-82 cm; Sample 153922B-3R-2, 39-44 cm; Sample 153-923A-12R-1, 30-38 cm; and Sample 153-923A-16R-3, 9-15 cm.

More evolved gabbros have lower modal olivine, higher modal clinopyroxene and, in some cases, orthopyroxene. The clinopyroxene typically occurs as brown cumulus grains, whereas olivine is more commonly an intercumulus phase. Again, texture and modal mineralogy are variable, but this group is typified by a preferred grain shape orientation, creating a fabric that may be in part magmatic. Layering is common, but it is usually produced by variations in grain size rather than by modal proportions of phases. Samples 153-921E2R-2, 18-24 cm, 153-923A-6R-1, 26-31 cm, and 153-923A-10R-2, $46-52 \mathrm{~cm}$, are from this group. Sample $153-924 \mathrm{~B}-2 \mathrm{R}-1,16-20 \mathrm{~cm}$, is transitional between the two groups, containing both green poikilitic and brown cumulus clinopyroxene. Oxide gabbros (i.e., those in which oxides form more than $5 \%$ of the mode) are a subset of this group. They usually occur in veins (e.g., Sample 153-920D-13R-2, $127-132 \mathrm{~cm}$ ) or associated with zones of high shear (e.g., Sample $153-922 \mathrm{~A}-2 \mathrm{R}-5,69-76 \mathrm{~cm}$ ).

As discussed in a previous section, systematic variations in modal mineralogy, texture, and inferred changes in mineral composition at Site 923 have been interpreted as evolutionary magmatic sequences or cycles. These cycles record a transition from primitive olivine gabbro or troctolite to a more evolved olivine gabbro or gabbro in which brown pyroxene occurs as a cumulus phase-essentially a transition from the first group to the second group described above. For this study, we analyzed two samples from one such magmatic cycle, Sample 153-923A-12R-1, 30-38 cm, and Sample 153-923A-10R-2, 46$52 \mathrm{~cm}$, which are from the bottom and top, respectively.

Two further samples were selected because of the interesting geologic problem they pose. At the bottom of Hole 920B, $0.74 \mathrm{~m}$ of amphibolitized microgabbro and gneissic gabbro was recovered. These two lithologies share a near vertical tectonic contact that runs along the entire length of the section. During shipboard analysis they were designated as a single unit because they appear to share the same high-temperature tectonite fabric. The amphibolite has a well-developed foliation and consists of recrystallized plagioclase, clinopyroxene, brown hornblende, olivine, and magnetite. The gneissic metagabbro has a coarse-grained porphyroclastic texture and contains an anhydrous mineral assemblage of clinopyroxene, plagioclase, and oxide minerals. The foliations in both are parallel and the contact is marked by an oxide-mineral-rich zone that is a few millimeters wide and subparallel to the foliation. Greenschist facies mineral assemblages in the gneissic gabbro are moderately abundant and contain only a trace of brown hornblende, whereas the amphibolite lacks retrograde minerals. The association of olivine, clinopyroxene and brown amphibole in near textural equilibrium with plagioclase, indicates formation at temperature of $\sim 600^{\circ}-800^{\circ} \mathrm{C}$ under low-pressure conditions for the amphibolite (Shipboard Scientific Party, 1995a). Two scenarios were proposed for this situation. The amphibolite and gneiss could originally have been formed during the same magmatic intrusion, but portions were subsequently altered and deformed independently. Alternatively, the two represent completely different magmatic events that responded differently to subsequent tectonic and alteration processes. Thus, in spite of the similar tectonite fabrics, the genetic relationship between the two lithologies is unclear.

\section{ANALYTICAL METHODS}

Most samples analyzed in this study are either visibly unaltered or contain only minor amounts of secondary alteration along grain boundaries or fractures (see Appendix). The most notable exceptions are Sample 153-920D-13R-2, 127-132 cm, in which the plagioclase has been totally replaced by prehnite, and Sample 153-920B-13R-4, $56-65 \mathrm{~cm}$, in which most of the clinopyroxene has been replaced by brown amphibole. Massif gabbros from Site 922 are also slightly more altered than those from other sites (i.e., $10 \%-20 \%$ total alteration), but they are significantly less altered than crosscutting gabbros from Site 920 . Because of the coarse grain size of most gabbroic samples, and because of the potential for isotopic disequilibrium between coexisting cumulate phases, all gabbros except the amphibolitized microgabbro were analyzed as mineral separates. 
Before crushing and isotopic analysis, all samples were washed in deionized (Milli-Q) water in an ultrasonic bath to remove any surface contaminants that might be acquired through handling or drilling. They were then hand-picked in methanol or ethanol using a binocular microscope to ensure the highest purity possible for each sample. All mineral separates, whole-rock powders, and basalt glasses were leached before dissolution in $6-\mathrm{M} \mathrm{HCl}$ on a hot plate at $\sim 100^{\circ} \mathrm{C}$ for $\sim 30 \mathrm{~min}$. The leachates were removed and the residue rinsed in Milli$\mathrm{Q} \mathrm{H}_{2} \mathrm{O}$. For some samples, the leachates and the final Milli-Q $\mathrm{H}_{2} \mathrm{O}$ rinses were combined and processed for isotopic analysis. Leached mineral residues were then crushed in an agate mortar before dissolution. Whole-rock powders were also analyzed in unleached form for comparative purposes.

Because the concentrations of $\mathrm{Pb}, \mathrm{Sr}$, and $\mathrm{Nd}$ are so low in oceanic gabbros, relatively large sample sizes (250-650 mg) were used. For total sample sizes greater than $250 \mathrm{mg}$, dissolutions were performed on multiple splits of the same sample, each split not exceeding 250 $\mathrm{mg}$ in weight. After dissolution using approximately $1 \mathrm{~mL}$ of $\mathrm{HNO}_{3}$ combined with $3-5 \mathrm{~mL}$ of $\mathrm{HF}$, residues were converted to nitrate and finally to chloride; $1 \mathrm{~mL}$ of $1-\mathrm{M} \mathrm{HBr}$ was then added to the residue. $\mathrm{Pb}$ was separated by passing the sample through columns prepared from PVP disposable pipette tips fitted with a 2-mm-diameter polyethylene frit and containing Dowex $1 \times 8$ 200-to 400-mesh resin. Completely new columns were prepared for each sample to minimize the $\mathrm{Pb}$ blank. The $\mathrm{Sr}$ and $\mathrm{Nd}$ fractions were collected in $2 \mathrm{~mL}$ of $1-\mathrm{M}$ $\mathrm{HBr}$ and $\mathrm{Pb}$ was collected in $1 \mathrm{~mL}$ of $6-\mathrm{M} \mathrm{HCl}$. Sr and $\mathrm{Nd}$ were purified using standard one- and two-column cation exchange techniques, respectively (Richards et al., 1976). For samples where multiple dissolutions were necessary, $\mathrm{Pb}$ and $\mathrm{Sr}$ column chemistries were performed for each split independently and the concentrated fraction of $\mathrm{Pb}$ and/or $\mathrm{Sr}$ combined at the end of the column procedure to avoid overloading the columns. The rare-earth element (REE) fractions extracted from the $\mathrm{Sr}$ column chemistry were combined before Nd column chemistry, and a single solution was put through the columns. $\mathrm{Sr}, \mathrm{Pb}$, and $\mathrm{Nd}$ were run as the metal species on single $\mathrm{Ta}$, single $\mathrm{Re}$, and double Re-Ta filaments, respectively, using a Finnigan MAT 262 multicollector mass spectrometer in static mode at the NERC Isotope Geosciences Laboratory (NIGL). Blanks for $\mathrm{Sr}, \mathrm{Nd}$, and $\mathrm{Pb}$ are typically less than $500 \mathrm{pg}, 800 \mathrm{pg}$, and $300 \mathrm{pg}$, respectively. Higher blanks were measured in a few cases (e.g., $14 \mathrm{ng}$ for $\mathrm{Sr}, 0.8$, and 3.8 $\mathrm{ng}$ for $\mathrm{Pb}$ ). However, we feel certain that these higher blanks are not indicative of the general blank level for several reasons: (1) two or more blanks were run with each batch of samples, and if one blank was high the others were usually low; (2) most samples have been run in duplicate, and the agreement between duplicates is excellent (Table 1); and (3) with the possible exception of $\mathrm{Pb}$, even the highest blanks measured were not sufficiently high to warrant blank correction. We therefore feel certain that none of the data reported here have been adversely affected by contamination during preparation. Nevertheless, we intend to reproduce an additional selection of samples from those batches where high blanks were encountered to confirm this.

${ }^{87} \mathrm{Sr} /{ }^{86} \mathrm{Sr}$ was normalized at run time to ${ }^{86} \mathrm{Sr} /{ }^{88} \mathrm{Sr}=0.1194 ;{ }^{143} \mathrm{Nd} /$ ${ }^{144} \mathrm{Nd}$ was similarly normalized to a value of ${ }^{146} \mathrm{Nd} /{ }^{144} \mathrm{Nd}=0.7219$. Samples for this study were analyzed over a period of 18 months; to minimize the effects of machine drift, sample results were further normalized to accepted values for NBS 987 and J\&M of 0.71024 and 0.51111 , respectively. Minimum uncertainties were derived from external precision of standard measurements that average $27 \mathrm{ppm}(1 \sigma)$ for ${ }^{143} \mathrm{Nd} /{ }^{144} \mathrm{Nd}$ and $18 \mathrm{ppm}(1 \sigma)$ for ${ }^{87} \mathrm{Sr} /{ }^{86} \mathrm{Sr}$. Based on repeated runs of NBS 981, the reproducibility of Pb-isotope measurements is better than $\pm 0.1 \%$ per a.m.u. $\mathrm{Pb}$-isotope ratios were corrected relative to the average standard $\mathrm{Pb}$ isotopic compositions of Todt et al. (1984).

Ratios of ${ }^{18} \mathrm{O} /{ }^{16} \mathrm{O}$ were also determined at NIGL. Oxygen was extracted using $\mathrm{ClF}_{3}$, with mineral separate powders left to react at $625^{\circ} \mathrm{C}$, and whole-rock powders at $525^{\circ} \mathrm{C}$ for $14 \mathrm{hr}$. Following the technique of Clayton and Mayeda (1963), oxygen from the fluorinat- ed samples was then converted to $\mathrm{CO}_{2}$. The collected $\mathrm{CO}_{2}$ gases were then analyzed on one of three ratio mass spectrometers (VG Sira Series II [Model Sira 10], Phoenix 390 [rebuilt VG model MM903], and VG Optima); the bulk of analyses were performed using the VG Sira Series II All final ${ }^{18} \mathrm{O} /{ }^{16} \mathrm{O}$ ratios have been reported as $\delta$ values in per mil (\%o), relative to the standard mean oceanic water (SMOW) standard of Craig (1961). All results are normalized to the accepted value (i.e., $+6.6 \%$ ) for the international standard XRC (Glendessary pyroxenite), a sample of which was run with each batch of analyses (average value $+6.2 \pm 0.4 \%$; $\mathrm{n}=25,2 \sigma$ ). In addition to the international standard, an internal check of sample reproducibility between runs was performed using mineral separate powders from Sample 153$921 \mathrm{~B}-4 \mathrm{R}-2,76-82 \mathrm{~cm}$. This produced average $\delta^{18} \mathrm{O}$ values of $+6.6 \pm$ $0.2 \%$ for plagioclase and $+5.8 \pm 0.1 \%$ for pyroxene $(2 \sigma ; n=4)$, with the errors falling within the acceptable range of $\pm 0.3 \%$. For all other samples, the analytical precision based on replicate analyses varied between \pm 0.1 and $\pm 0.3 \% \circ(2 \sigma)$.

\section{RESULTS $\mathrm{Sr}, \mathrm{Nd}, \mathrm{Pb}$ Isotopes}

Results of isotopic analysis are listed in Tables 1 and 2 and are shown graphically in Figures 2-5. Considered collectively, there is a relatively wide range of $\mathrm{Sr}$ - and $\mathrm{Nd}$-isotope values for the samples analyzed, but the different lithologic groups cluster into more restricted compositional ranges. Mineral separates from massif gabbros (Sites 921-924) have relatively high Nd- and low Sr-isotope values. With few exceptions, variations in the average ${ }^{143} \mathrm{Nd} /{ }^{144} \mathrm{Nd}$ ratios are within analytical uncertainty as determined by standard reproducibility. However, clinopyroxenes (cpx) from Site 922 gabbros have slightly higher ${ }^{87} \mathrm{Sr} /{ }^{86} \mathrm{Sr}$ than both massif gabbros from other sites $\left(\Delta_{\text {cpx-avg }}=6-12 \times 10^{-5} ; \mathrm{avg}=\right.$ average $)$ and plagioclase (plag) from the same sample $\left(\Delta_{\text {cpx-plag }}=6-7 \times 10^{-5}\right)$ (Table 1$)$, compared with an analytical reproducibility of the standard of $2.5 \times 10^{-5}(2 \sigma)$. Diabase dikes that crosscut the massif gabbros in Holes 921B and 921C lie within the field defined by the massif gabbros, overlapping the higher ${ }^{87} \mathrm{Sr} /{ }^{86} \mathrm{Sr}$ values of clinopyroxenes from Site 922 . Both the gabbros and dikes from Sites 921 to 924 lie toward the depleted end of the field for North Atlantic N-MORB (normal MORB), but they have slightly lower Nd-isotope ratios than those reported by Machado et al. (1982) for samples dredged from the southern wall of the Kane Fracture Zone approximately $5 \mathrm{~km}$ to the north. Note that these values are among the highest reported for North Atlantic MORB, the only higher values being two unusually depleted samples from $17^{\circ} \mathrm{N}$ (Dosso et al., 1993). A value for BCR rock standard was reported by Machado et al. (1982) with this data set and it has a significantly higher value than BCR analyzed in the NIGL laboratory. We have normalized their data using these values for Figure 2. Even so, the values remain higher than any reported for the Kane Fracture Zone area. We have also analyzed plagiogranite and diabase (P.D. Kempton, unpubl. data) dredged from localities near those sampled and analyzed by Machado et al. (1982), and these samples yield Nd-isotope values identical to those of Leg 153 gabbros. We therefore suspect that there is an interlaboratory bias and that the Machado et al. (1982) data may need revision to be internally consistent with our data.

Gabbroic dikes from Site 920 have similar Nd-isotope compositions to massif gabbros from Sites 921 to 924 , but they have significantly higher ${ }^{87} \mathrm{Sr} /{ }^{86} \mathrm{Sr}$ ratios. This is consistent with the significantly greater degree of alteration of these samples (see Appendix). In contrast, the amphibolitized microgabbro from the bottom of Hole 920B has a distinctly lower $\mathrm{Nd}$-isotope composition than any gabbroic sample analyzed in this study; its $\mathrm{Sr}$-isotope composition is intermediate between those of the massif gabbros (Sites 921-924) and diabase dikes from Site 920 . Its composition contrasts markedly with the coarse-grained gneissic gabbro with which it is in contact, the latter being indistinguishable from other gabbros from the area (Table 1). 
Table 1. Sr-, Nd-, $\mathrm{Pb}$-, and $\mathrm{O}$-isotope compositions of Leg 153 gabbros.

\begin{tabular}{|c|c|c|c|c|c|c|c|c|c|c|c|c|c|}
\hline $\begin{array}{l}\text { Core, section, } \\
\text { interval }(\mathrm{cm})\end{array}$ & & Type & ${ }^{87} \mathrm{Sr} /{ }^{86} \mathrm{Sr}$ & $\begin{array}{l}{ }^{87} \mathrm{Sr} /{ }^{86} \mathrm{Sr} \\
\text { average }\end{array}$ & ${ }^{143} \mathrm{Nd} /{ }^{144} \mathrm{Nd}$ & $\begin{array}{c}{ }^{143} \mathrm{Nd} /{ }^{144} \mathrm{Nd} \\
\text { average }\end{array}$ & ${ }^{206} \mathrm{~Pb} /{ }^{204} \mathrm{~Pb}$ & ${ }^{207} \mathrm{~Pb} /{ }^{204} \mathrm{~Pb}$ & ${ }^{208} \mathrm{~Pb} / 204 \mathrm{~Pb}$ & $\begin{array}{c}{ }^{206} \mathrm{~Pb} /{ }^{204} \mathrm{~Pb} \\
\text { average }\end{array}$ & $\begin{array}{c}{ }^{207} \mathrm{~Pb} /{ }^{204} \mathrm{~Pb} \\
\text { average }\end{array}$ & $\begin{array}{c}{ }^{208} \mathrm{~Pb} /{ }^{004} \mathrm{~Pb} \\
\text { average }\end{array}$ & $\delta^{18} \mathrm{O}$ \\
\hline \multicolumn{14}{|l|}{ 153-920B- } \\
\hline $\begin{array}{l}13 R-4,56-65 \\
13 R-4,56-65\end{array}$ & $\begin{array}{l}\text { (1A) } \\
\text { (1A) }\end{array}$ & $\begin{array}{l}\text { Plg LR } \\
\text { Plg LR }\end{array}$ & $\begin{array}{l}0.703329 \\
0.703299\end{array}$ & 0.703314 & 0.513200 & 0.513200 & 18.141 & 15.490 & 37.583 & 18.141 & 15.490 & 37.583 & 12.0 \\
\hline $13 R-4,56-65$ & (1A) & Plg LL & 0.704576 & 0.704576 & 0.513215 & 0.513215 & 18.235 & 15.488 & 37.647 & 18.235 & 15.488 & 37.647 & \\
\hline $13 R-4,30-36$ & (1A) & AM LR & 0.702618 & 0.702606 & 0.513084 & 0.513069 & 18.815 & 15.531 & 38.192 & 18.772 & 15.540 & 38.215 & 5.6 \\
\hline $13 R-4,30-36$ & (1B) & AM LR & 0.702612 & & 0.513054 & & 18.728 & 15.548 & 38.238 & & & & \\
\hline $13 R-4,30-36$ & (IC) & AM LR & 0.702587 & & & & & & & & & & \\
\hline $13 R-4,30-36$ & (1A) & AM UL & 0.702579 & 0.702612 & & 0.513062 & 18.691 & 15.574 & 38.318 & 18.667 & 15.567 & 38.281 & \\
\hline $13 R-4,30-36$ & (1B) & AM UL & 0.702627 & & 0.513064 & & 18.643 & 15.560 & 38.244 & & & & \\
\hline $13 R-4,30-36$ & (1C) & AM UL & 0.702631 & & 0.513060 & & & & & & & & \\
\hline $13 \mathrm{R}-4,30-36$ & (1C) & AM LL* & 0.702742 & 0.702742 & 0.513069 & 0.513069 & & & & & & & \\
\hline $13 \mathrm{R}-4,30-36$ & (1A) & AM LL & 0.702636 & 0.702648 & 0.513094 & 0.513066 & 18.544 & 15.576 & 38.332 & 18.520 & 15.580 & 38.339 & \\
\hline $13 R-4,30-36$ & (1A) & AM LL & 0.702664 & & & & & & & & & & \\
\hline $13 \mathrm{R}-4,30-36$ & (1B) & AM LL & 0.702671 & & 0.513065 & & 18.497 & 15.583 & 38.346 & & & & \\
\hline $13 R-4,30-36$ & (1C) & AM LL & 0.702621 & & 0.513038 & & & & & & & & \\
\hline 153-920D- & & & & & & & & & & & & & \\
\hline $13 \mathrm{R}-2,127-132$ & (IA) & Preh/plg & 0.703581 & 0.703588 & & 0.513213 & 18.205 & 15.481 & 37.622 & 18.190 & 15.466 & 37.578 & 6.3 \\
\hline 13R-2, 127-132 & (1A) & Preh/plg & 0.703588 & & & & & & & & & & \\
\hline 13R-2, 127-132 & (IB) & Preh/plg & 0.703585 & & 0.513213 & & 18.175 & 15.451 & 37.535 & & & & \\
\hline 13R-2, 127-132 & (1B) & Preh/plg & 0.703595 & & & & & & & & & & \\
\hline 13R-2, 127-132 & (1A) & Pyx LR & 0.702752 & 0.702742 & 0.513187 & 0.513190 & 18.165 & 15.480 & 37.587 & 18.210 & 15.512 & 37.659 & 5.3 \\
\hline $\begin{array}{l}13 R-2,127-132 \\
13 R-2,127-132\end{array}$ & (IA) & Pyx LR & 0.702741 & & & & & & & & & & \\
\hline $\begin{array}{l}13 \mathrm{R}-2,127-132 \\
13 \mathrm{R}-2,127-132\end{array}$ & $\begin{array}{l}\text { (IB) } \\
\text { (1B) }\end{array}$ & $\begin{array}{l}\text { Pyx LR } \\
\text { Pyx LR }\end{array}$ & $\begin{array}{l}0.702746 \\
0.702727\end{array}$ & & 0.513194 & & 18.254 & 15.543 & 37.732 & & & & \\
\hline $13 \mathrm{R}-2,127-132$ & (1A) & Preh/plg LL & 0.705332 & 0.705332 & & & & & & & & & \\
\hline $13 \mathrm{R}-2,127-132$ & (1A) & Pyx LL & 0.705485 & 0.705485 & & & 18.187 & 15.517 & 37.689 & 18.187 & 15.517 & 37.689 & \\
\hline 153-921B- & & & & & & & & & & & & & \\
\hline $4 \mathrm{R}-2,76-82$ & (1A) & Plg LR & 0.702359 & 0.702362 & 0.513192 & 0.513176 & 18.111 & 15.488 & 37.569 & 18.159 & 15.468 & 37.557 & 6.5 \\
\hline $4 \mathrm{R}-2,76-82$ & (1A) & Plg LR & 0.702359 & & & & & & & & & & \\
\hline $4 \mathrm{R}-2,76-82$ & (1B) & Plg LR & 0.702367 & & 0.513160 & & 18.206 & 15.447 & 37.544 & & & & \\
\hline $4 \mathrm{R}-2,76-82$ & (1B) & Plg LR & 0.702360 & & & & & & & & & & \\
\hline $4 R-2,76-82$ & (1A) & Pyx LR & 0.702361 & 0.702369 & 0.513220 & 0.513220 & 18.050 & 15.450 & 37.424 & & & & 5.8 \\
\hline $4 \mathrm{R}-2,76-82$ & (1A) & Pyx LR & 0.702379 & & & & & & & & & & \\
\hline $4 \mathrm{R}-2,76-82$ & (1B) & Pyx LR & 0.702366 & & 0.513220 & & 18.050 & 15.450 & 37.424 & & & & \\
\hline $4 \mathrm{R}-2,76-82$ & (1A) & Pig LL & 0.702427 & 0.702427 & & & 18.024 & 15.576 & 37.724 & 18.024 & 15.576 & 37.724 & \\
\hline $4 R-2,76-82$ & (1A) & Pyx LL & 0.702503 & 0.702503 & & & 17.863 & 15.536 & 37.356 & 17.863 & 15.536 & 37.356 & \\
\hline $153-921 \mathrm{E}-$ & & & & & & & & & & & & & \\
\hline $2 \mathrm{R}-2,18-24$ & (1A) & Plg LR & 0.702358 & 0.702344 & 0.513197 & 0.513204 & 18.043 & 15.456 & 37.462 & 18.053 & 15.458 & 37.460 & 5.9 \\
\hline $2 \mathrm{R}-2,18-24$ & (1A) & Plg LR & 0.702319 & & & & & & & & & & \\
\hline $2 \mathrm{R}-2,18-24$ & (1A) & Plg LR & 0.702337 & & & & & & & & & & \\
\hline $2 \mathrm{R}-2,18-24$ & (1B) & Plg LR & 0.702361 & & 0.513211 & & 18.062 & 15.459 & 37.458 & & & & \\
\hline $2 \mathrm{R}-2,18-24$ & (1B) & Plg LR & 0.702345 & & & & & & & & & & \\
\hline $2 \mathrm{R}-2,18-24$ & (1A) & Pyx LR & 0.702390 & 0.702378 & 0.513174 & 0.513182 & 18.044 & 15.435 & 37.413 & & & & 5.7 \\
\hline $2 \mathrm{R}-2,18-24$ & (1A) & Pyx LR & 0.702352 & & & & & & & & & & \\
\hline $2 \mathrm{R}-2,18-24$ & (1A) & Pyx LR & 0.702366 & & & & & & & & & & \\
\hline $2 \mathrm{R}-2,18-24$ & (1A) & Pyx LR & 0.702331 & & & & & & & & & & \\
\hline $2 \mathrm{R}-2,18-24$ & (IB) & Pyx LR & 0.702406 & & 0.513191 & & 18.044 & 15.435 & 37.413 & & & & \\
\hline $2 \mathrm{R}-2,18-24$ & (IB) & Pyx LR & 0.702420 & & & & & & & & & & \\
\hline $2 \mathrm{R}-2,18-24$ & (1A) & Pig LL & 0.704511 & 0.704511 & & & 18.144 & 15.590 & 37.914 & 18.144 & 15.590 & 37.914 & \\
\hline $2 \mathrm{R}-2,18-24$ & (1A) & Pyx LL & 0.708404 & 0.708404 & & & 18.150 & 15.572 & 37.824 & 18.150 & 15.572 & 37.824 & \\
\hline $153-922 \mathrm{~A}-$ & & & & & & & & & & & & & \\
\hline 2R-5, 69-76 & (1A) & PIg LR & 0.702401 & 0.702403 & 0.513186 & 0.513186 & 18.104 & 15.468 & 37.520 & 18.104 & 15.468 & 37.520 & 6.7 \\
\hline $2 R-5,69-76$ & (1A) & Plg LR & 0.702405 & & & & & & & & & & \\
\hline $2 R-5,69-76$ & (IA) & Pyx LR & 0.702451 & 0.702476 & 0.513217 & 0.513217 & 18.116 & 15.471 & 37.516 & 18.116 & 15.471 & 37.516 & 5.4 \\
\hline & (1A) & Pyx LR & 0.702501 & 0.702373 & & & & & & & & & \\
\hline $\begin{array}{l}\text { 2R-5, 69-76 } \\
2 R-5,69-76\end{array}$ & (1A) & Plg LL & 0.702373 & & & & 18.113 & 15.544 & 37.681 & 18.113 & 15.544 & 37.681 & \\
\hline $2 \mathrm{R}-5,69-76$ & (1A) & Pyx LL & 0.703990 & 0.703990 & & & 18.203 & 15.516 & 37.684 & 18.203 & 15.516 & 37.684 & \\
\hline $\begin{array}{l}153-922 \mathrm{~B}- \\
3 \mathrm{R}-2,39-44\end{array}$ & & & & & & & & & & & & & \\
\hline $\begin{array}{l}3 R-2,39-44 \\
3 R-2,39-44\end{array}$ & (1A) & Plg LR & 0.702352 & 0.702360 & 0.513210 & 0.513194 & 18.053 & 15.454 & 37.436 & 18.053 & 15.454 & 37.436 & 6.0 \\
\hline $\begin{array}{l}3 \mathrm{R}-2,39-44 \\
3 \mathrm{R}-2,39-44\end{array}$ & (1A) & Plg LR & 0.702358 & & & & & & & & & & \\
\hline $\begin{array}{l}3 \mathrm{R}-2,39-44 \\
3 \mathrm{R}-2,39-44\end{array}$ & $(2 \mathrm{~A})$ & Plg LR & 0.702369 & & 0.513178 & & 18.004 & 15.409 & 37.392 & & & & \\
\hline $3 R-2,39-44$ & $(2 \mathrm{~A})$ & Plg LR & 0.702372 & & & & & & & & & & \\
\hline 3R-2, 39-44 & $(2 \mathrm{~A})$ & Plg LR & 0.702345 & & & & & & & & & & \\
\hline 3R-2, 39-44 & (1A) & Pyx LR & 0.702388 & 0.702421 & 0.513218 & 0.513203 & 18.078 & 15.449 & 37.469 & 18.116 & 15.471 & 37.553 & 5.5 \\
\hline $3 R-2,39-44$ & (1A) & Pyx LR & 0.702448 & & & & & & & & & & \\
\hline $3 R-2,39-44$ & (1B) & Pyx LR & 0.702439 & & 0.513187 & & 18.116 & 15.471 & 37.553 & & & & \\
\hline $3 R-2,39-44$ & (1B) & Pyx LR & 0.702430 & & & & & & & & & & \\
\hline $3 R-2,39-44$ & (IB) & Pyx LR & 0.702415 & & & & & & & & & & \\
\hline $3 R-2,39-44$ & (1B) & Pyx LR & 0.702402 & & & & & & & & & & \\
\hline $3 R-2,39-44$ & (1A) & Plg LL & 0.702583 & 0.702583 & 0.513184 & 0.513184 & 18.366 & 15.566 & 37.983 & 18.366 & 15.566 & 37.983 & \\
\hline $3 R-2,39-44$ & (1A) & Pyx LL & 0.705030 & 0.705030 & 0.513203 & 0.513203 & 18.161 & 15.541 & 37.733 & 18.161 & 15.541 & 37.733 & \\
\hline $153-923 \mathrm{~A}-$ & & & & & & & & & & & & & \\
\hline $\begin{array}{l}6 \mathrm{R}-1,26-31 \\
6 \mathrm{R}-1,26-31\end{array}$ & $\begin{array}{l}\text { (1A) } \\
\text { (1A) }\end{array}$ & $\begin{array}{l}\text { Plg LR } \\
\text { Plg LR }\end{array}$ & $\begin{array}{l}0.702344 \\
0.702346\end{array}$ & 0.702345 & 0.513213 & 0.513213 & 18.039 & 15.450 & 37.429 & 18.039 & 15.450 & 37.429 & 6.3 \\
\hline $6 \mathrm{R}-1,26-31$ & (1A) & Pyx LR & & 0.702339 & 0.513213 & 0.513213 & 18.063 & 15.471 & 37.505 & 18.063 & 15.471 & 37.505 & 5.6 \\
\hline $6 \mathrm{R}-1,26-31$ & (1A) & Pyx LR & 0.702337 & & & & & & & & & & \\
\hline $6 \mathrm{R}-1,26-31$ & (1A) & Pyx LR & 0.702340 & & & & & & & & & & \\
\hline $10 \mathrm{R}-2,46-52$ & (1A) & Plg LR & 0.702345 & 0.702349 & 0.513208 & 0.513180 & 18.020 & 15.442 & 37.411 & 18.054 & 15,461 & 37.473 & 6.1 \\
\hline $10 \mathrm{R}-2,46-52$ & (1A) & Plg LR & 0.702333 & & & & & & & & & & \\
\hline $10 \mathrm{R}-2,46-52$ & (1B) & Plg LR & 0.702353 & & 0.513152 & & 18.087 & 15.480 & 37.534 & & & & \\
\hline $10 \mathrm{R}-2,46-52$ & (1B) & Plg LR & 0.702364 & & & & & & & & & & \\
\hline $10 \mathrm{R}-2,46-52$ & (1A) & BPуyx LR & 0.702346 & 0.702357 & 0.513216 & 0.513198 & 17.828 & 15.587 & 37.476 & 17.924 & 15.524 & 37.474 & \\
\hline $10 \mathrm{R}-2,46-52$ & (1A) & GPyx LR & 0.702345 & & 0.513198 & & 18.020 & 15.461 & 37.472 & & & & \\
\hline $10 \mathrm{R}-2,46-52$ & (1A) & GPyx LR & 0.702350 & & & & & & & & & & \\
\hline $10 \mathrm{R}-2,46-52$ & $(2 \mathrm{~A})$ & BGPyx LR & 0.702367 & & 0.513180 & & & & & & & & 5.8 \\
\hline
\end{tabular}


Table 1 (continued).

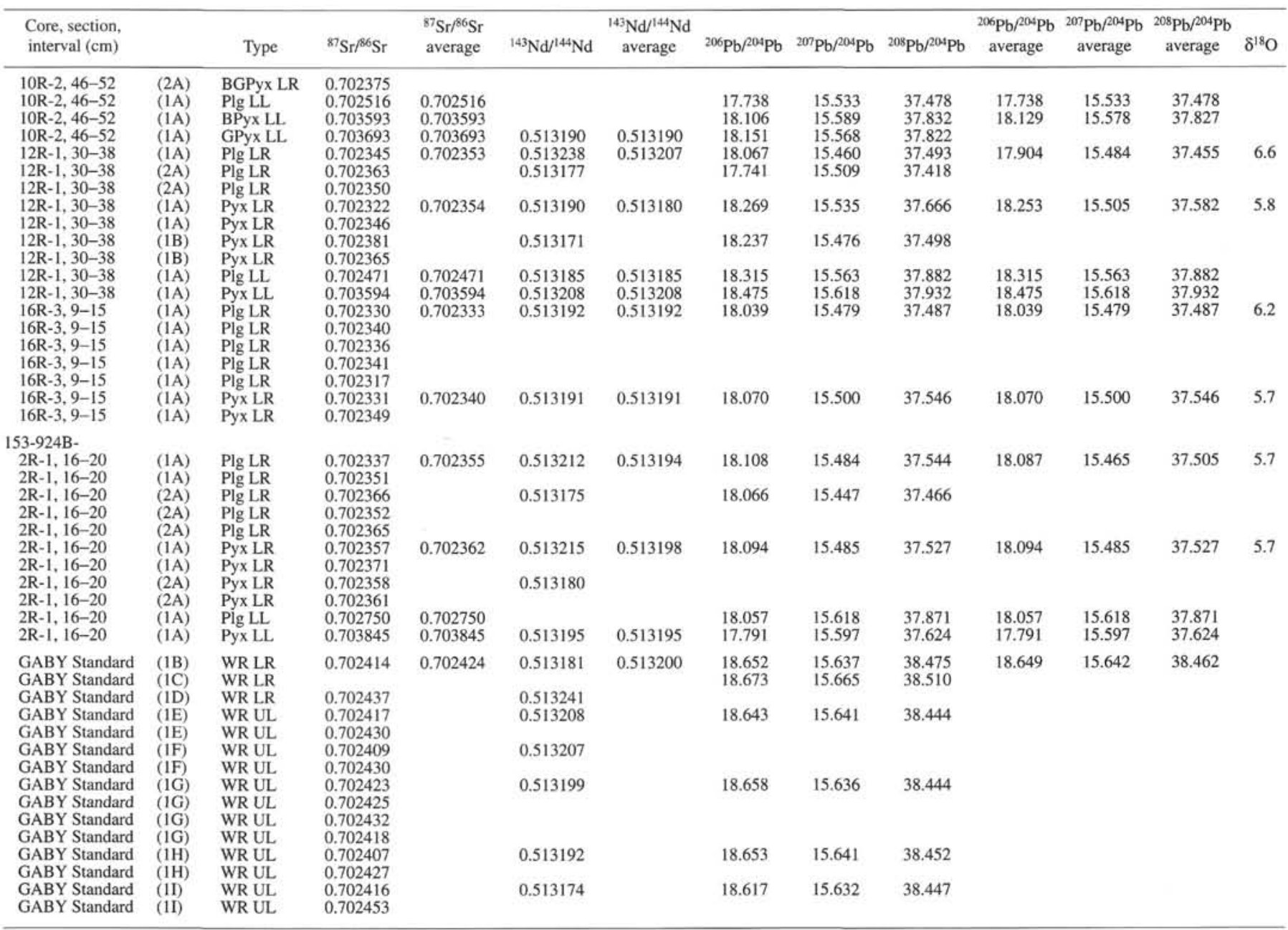

Notes: Abbreviations as follows: $\mathrm{plg}=$ plagioclase, $\mathrm{pyx}=$ pyroxene, preh $=$ prehnite, $\mathrm{AM}=$ amphibolitized microgabbro, $\mathrm{WR}=$ whole rock, $\mathrm{LR}=$ leached residue, $\mathrm{LL}=$ leachate, and $\mathrm{UL}=$ unleached; ${ }^{*}=1.5 \mathrm{M} \mathrm{HCl}$ leach (all other leachates are $6 \mathrm{M} \mathrm{HCl}$ ). Numbers in brackets after sample name indicate sample split and order of dissolution (i.e., $1=$ first picking and dissolution of the sample, 2 = same sample, but a completely new dissolution using freshly picked minerals). Letters indicate order of multiple analyses from the same sample split. Multiple loadings for mass spectrometry from the same dissolution ( $\mathrm{Sr}$ only) carry the same letter, but a subsequent dissolution from the same hand-picked mineral split or rock powder is indicated by a different letter.

Table 2. Sr-, Nd-, and Pb-isotope compositions of recent MARK basalts.

\begin{tabular}{|c|c|c|c|c|c|}
\hline Sample & ${ }^{87} \mathrm{Sr} /{ }^{86} \mathrm{Sr}$ & ${ }^{143} \mathrm{Nd} /{ }^{144} \mathrm{Nd}$ & ${ }^{206} \mathrm{~Pb} / 204 \mathrm{~Pb}$ & ${ }^{20} \mathrm{~Pb} /{ }^{\circ-5} \mathrm{~Pb}$ & ${ }^{200} \mathrm{~Pb} /{ }^{204} \mathrm{~Pb}$ \\
\hline TK 11-2 & 0.702326 & 0.513183 & 18.459 & 15.517 & 37.863 \\
\hline $\begin{array}{l}\text { TK 13-11(a) } \\
\text { TK 13-11(b) }\end{array}$ & 0.702341 & $\begin{array}{l}0.513161 \\
0.513175\end{array}$ & 18.369 & 15.543 & 37.899 \\
\hline TK 15-2 (a) & 0.702389 & 0.513143 & 18.739 & 15.584 & 38.194 \\
\hline TK $15-2$ (b) & 0.702366 & 0.513175 & & & \\
\hline TK 16 (a) & 0.702335 & 0.513153 & 18.477 & 15.545 & 37.971 \\
\hline TK 16 (b) & & 0.513156 & & & \\
\hline TK $18-2$ (a) & 0.702379 & 0.513149 & & & \\
\hline TK $18-2$ (b) & & 0.513152 & & & \\
\hline TK 18-3 (a) & 0.702339 & 0.513144 & 18.388 & 15.504 & 37.806 \\
\hline TK 18-3 (b) & & 0.513149 & 10 - 70 & 1519 & \\
\hline $\begin{array}{l}\text { TK 20-2 (a) } \\
\text { TK 20-2 (b) }\end{array}$ & $\begin{array}{l}0.702344 \\
0.702350\end{array}$ & $\begin{array}{l}0.513152 \\
0.513169\end{array}$ & 18.278 & 15.486 & 37.692 \\
\hline TK 25 (a) & 0.702352 & 0.513157 & 18.298 & 15.485 & 37.723 \\
\hline TK 25 (b) & & 0.513148 & & & \\
\hline
\end{tabular}

Note: Letters in parentheses after the same name indicate multiple analyses from the same dissolution.
This indicates that the two lithologies record distinct intrusive events and that the deformation was later superimposed on both.

The diabase dikes from Site 920 are isotopically distinct, having high ${ }^{87} \mathrm{Sr} /{ }^{86} \mathrm{Sr}$ - and $\mathrm{Nd}$-isotope values intermediate between those of the massif gabbros (Sites 921-924) and the amphibolitized microgabbro from Site 920 . Recent basalts from the neovolcanic zone have low ${ }^{87} \mathrm{Sr} /{ }^{86} \mathrm{Sr}$, similar to the gabbros and diabase dikes from MARK, but most have lower ${ }^{143} \mathrm{Nd} /{ }^{144} \mathrm{Nd}$ ratios when compared with the MARK gabbros (Fig. 2).

Pyroxenes separated from Site 920 ultramafic peridotites have the most depleted Nd-isotope signatures found within the MARK area to date (Kempton and Stephens, this volume) and overlap the high end of the range of values reported for North Atlantic MORB, as well as the basalts from the southern wall of the Kane Fracture Zone (Machado et al., 1982). The high Sr-isotope composition clearly indicates seawater alteration, and it is unlikely that a primary Sr-isotope signature can be derived from the material because of the extensive interaction with seawater during serpentinization (Kempton and Stephens, this volume).

Leachates from gabbro mineral separates exhibit similar Nd-isotope compositions to the leached residues, but the $\mathrm{Sr}$-isotope compositions are significantly higher (Fig. 3). Furthermore, leachates ex- 


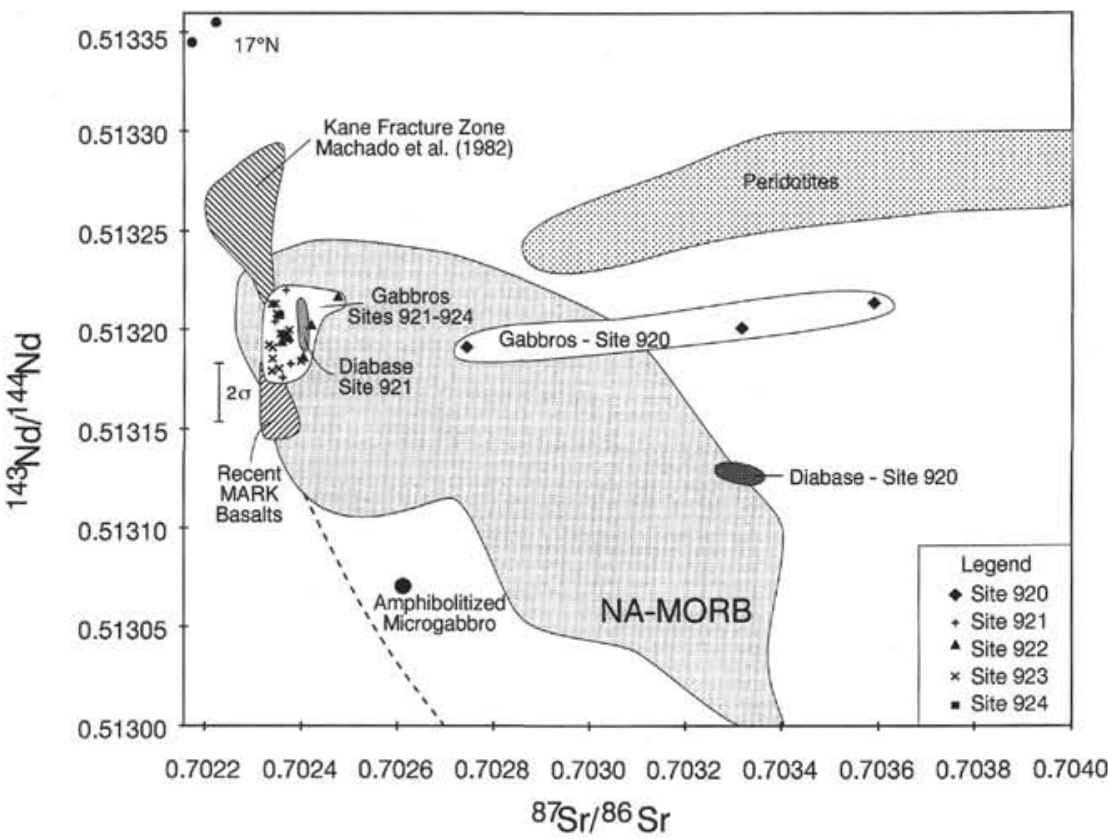

Figure 2. Plot of ${ }^{87} \mathrm{Sr} /{ }^{86} \mathrm{Sr}$ vs. ${ }^{143} \mathrm{Nd} /{ }^{144} \mathrm{Nd}$ for Leg 153 gabbros. Data plotted are average compositions from Table 1. Shown for comparison are fields for peridotites from Site 920 (Kempton and Stephens, this volume), diabase dikes from Sites 920 and 921 (Kempton and Casey, this volume), amphibolitized microgabbro from Site 920 , recent MARK basalt glasses from the neovolcanic zone, and North Atlantic N-MORB (NA-MORB). Data for NAMORB field from Cohen et al. (1980), Cohen and O'Nions (1982), Dosso et al. (1993), Frey et al. (1993), Ito et al. (1987), and White and Hofmann (1982). Data from Machado et al. (1982) are shown separately; these data have been normalized using their published value for BCR $(0.512679 \pm 20)$ to make them consistent with NIGL measured values for this rock standard (0.512602). Also shown separately are the two unusual samples from $17^{\circ} \mathrm{N}$ (Dosso et al., 1993). Data from the $15^{\circ} 20^{\prime}$ Fracture Zone (Dosso et al., 1991) have been excluded from the shaded field for NA-MORB; dashed line indicates shape of MORB field if these data were included. ${ }^{143} \mathrm{Nd} /{ }^{144} \mathrm{Nd} 2 \sigma$ is based on replicate analyses of J\&M Nd standard solution; $2 \sigma$ for ${ }^{87} \mathrm{Sr} /{ }^{86} \mathrm{Sr}$ is within the size of the symbols.

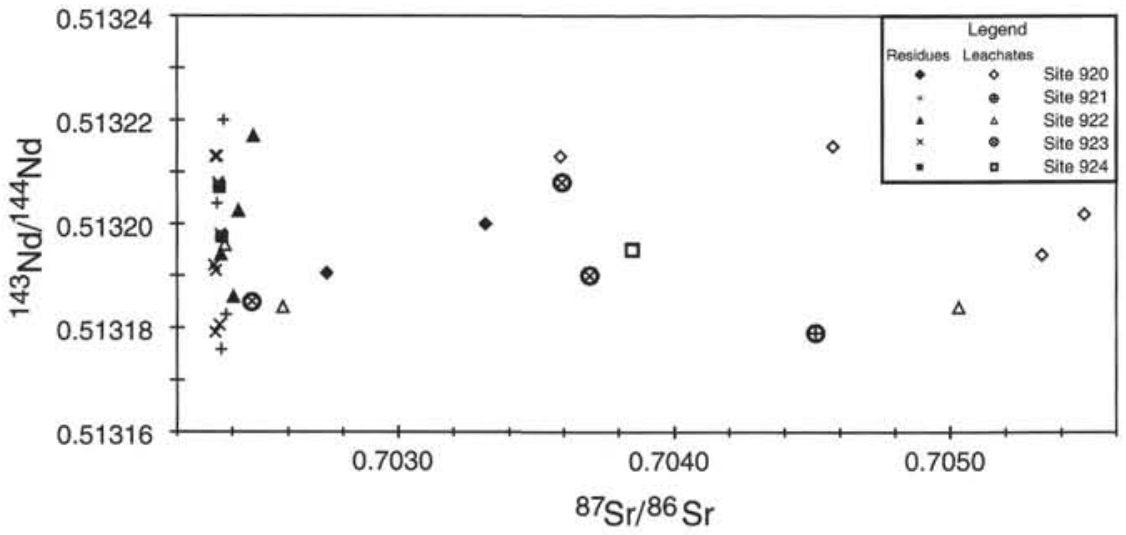

Figure 3. Expanded view of ${ }^{87} \mathrm{Sr} /{ }^{86} \mathrm{Sr}$ vs. ${ }^{143} \mathrm{Nd} /{ }^{144} \mathrm{Nd}$ for Leg 153 gabbros, including the compositions of leachates extracted from mineral separates. Data plotted are average compositions from Table 1 . tracted from clinopyroxenes have consistently higher ${ }^{87} \mathrm{Sr} /{ }^{86} \mathrm{Sr}$ values than those extracted from the coexisting plagioclase (Fig. 4A). There are two probable explanations for this, either or both of which may apply. First, the $\mathrm{Sr}$ concentration in cumulate clinopyroxenes is lower than that in plagioclase by about an order of magnitude and thus interaction with $\mathrm{Sr}$-rich hydrothermal fluids will register more quickly (i.e., at lower water/rock ratios) in the clinopyroxene. In addition, the clinopyroxenes are susceptible to replacement by secondary phases, such as amphibole, which can be difficult to separate during preparation of the mineral separates. Therefore, the clinopyroxene separates analyzed may be inherently more altered than the plagioclase.

Figure 5 summarizes the $\mathrm{Pb}$-isotope variations in the MARK area. In ${ }^{206} \mathrm{~Pb} /{ }^{204} \mathrm{vs} .{ }^{207} \mathrm{~Pb} /{ }^{204} \mathrm{~Pb}$, the data collectively form a linear array, in which all but the peridotites fall within the field of N-MORB. However, similar to $\mathrm{Sr}-\mathrm{Nd}$ systematics, distinct groups can be identified. In most cases, the massif gabbros exhibit a relatively restricted range of values which lie at the low end of the N-MORB field, above and parallel to the Northern Hemisphere Reference Line (NHRL). Gabbros from Site 923 , however, exhibit a wider range of ${ }^{206} \mathrm{~Pb} /{ }^{204} \mathrm{~Pb}$ values than samples from other holes. In particular, clinopyroxene from Sample $153-923 \mathrm{~A}-12 \mathrm{R}-1,30-38 \mathrm{~cm}$, has the highest ${ }^{206} \mathrm{~Pb} /{ }^{204} \mathrm{~Pb}$ of any massif gabbro, whereas the coexisting plagioclase from this sample has the least radiogenic composition (Table 1). Similarly, clinopyroxene from Sample 153-923A-10R-2, 46-52 cm, approximately $8 \mathrm{~m}$ above, has an unradiogenic ${ }^{206} \mathrm{~Pb} /{ }^{204} \mathrm{~Pb}$ ratio like that of the pla- gioclase from Sample 153-923-12R-1, 30-38 cm. These two samples with unradiogenic ${ }^{206} \mathrm{~Pb} / 204 \mathrm{~Pb}$ values are also distinguished by their high $\Delta 7 / 4$ (i.e., they have higher ${ }^{207} \mathrm{~Pb} /{ }^{204} \mathrm{~Pb}$ for a given ${ }^{206} \mathrm{~Pb} /{ }^{204} \mathrm{~Pb}$ than would be predicted by the NHRL). These samples have been analyzed in duplicate and the different analyses agree well (Table 1), so we are confident that these results are "real" and not caused by analytical uncertainty.

Analogous to $\mathrm{Sr}$-isotope systematics, ${ }^{206} \mathrm{~Pb} /{ }^{204} \mathrm{~Pb}$ values are slightly higher for samples from Site 922 than other massif gabbros and, with the exception of the one pyroxene from Site 923A (Table 1), gabbro dikes from Site 920 have higher ${ }^{206} \mathrm{~Pb} /{ }^{204} \mathrm{~Pb}$ than do the massif gabbros (Fig. 5A). The most radiogenic ${ }^{206} \mathrm{~Pb} / 204 \mathrm{~Pb}$ value, however, is found in the amphibolitized microgabbro, a result consistent with its unique composition in $\mathrm{Sr}-\mathrm{Nd}$ space.

The diabase dikes from Site 921 are similar to the massif gabbros in their $\mathrm{Pb}$-isotope composition, but the dikes from Site 920 are significantly more radiogenic. Recent basalts from the neovolcanic zone exhibit a relatively large range of values that extends from the field of massif gabbros to the radiogenic composition of the amphibolite.

Leachates show a distinctly larger range of compositions than the associated mineral separate residues, extending to both higher and lower ${ }^{206} \mathrm{~Pb} /{ }^{204} \mathrm{~Pb}$ ratios (Fig. 5). Most striking, however, is the predominance of high ${ }^{207} \mathrm{~Pb} / 204 \mathrm{~Pb}$ ratios. Two additional points are worthy of note. The leachates from gabbroic dikes (Site 920) do not show these higher ${ }^{207} \mathrm{~Pb} /{ }^{204} \mathrm{~Pb}$ ratios, and are instead similar to the compo- 

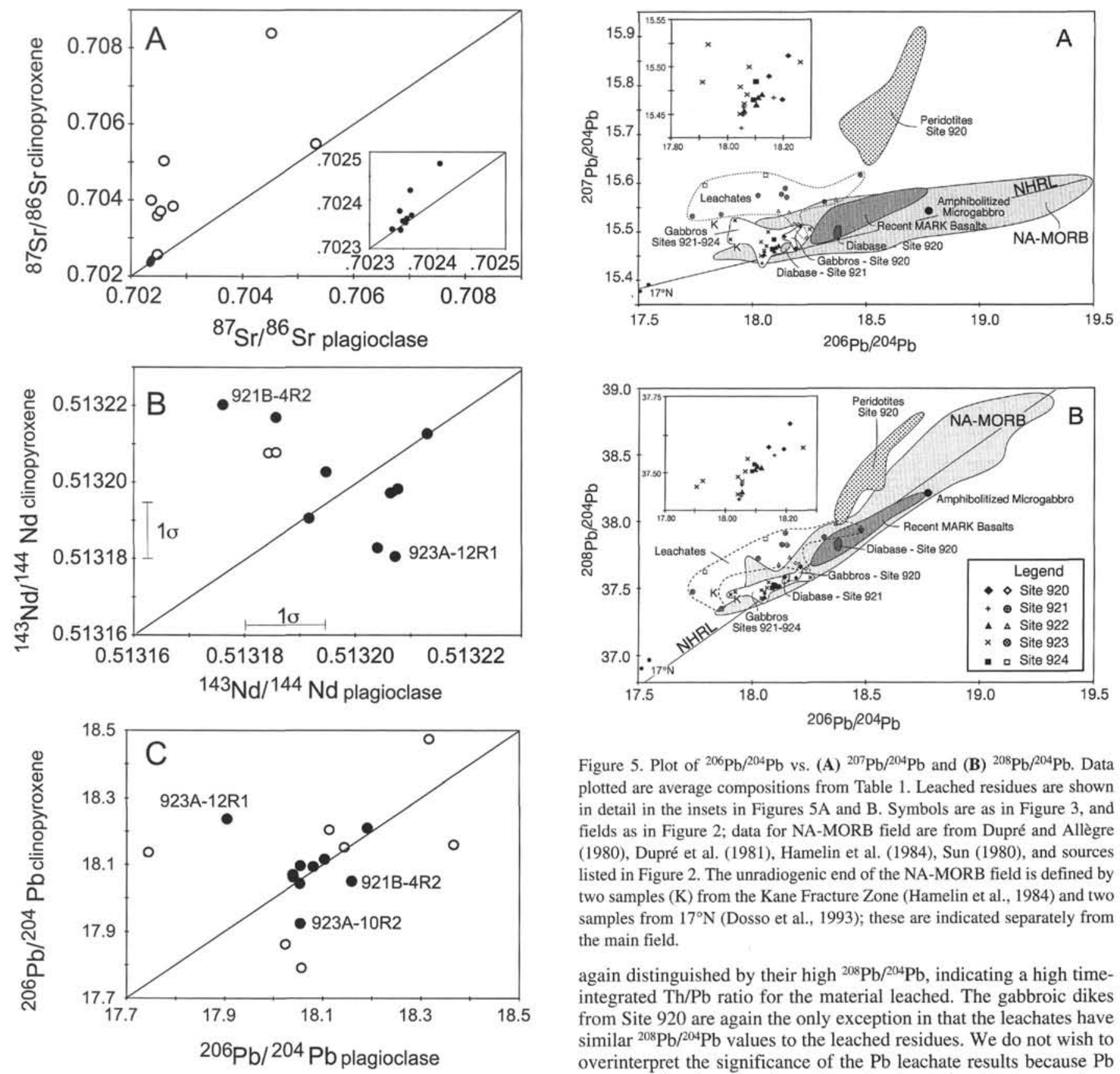

Figure 5. Plot of ${ }^{206} \mathrm{~Pb} /{ }^{204} \mathrm{~Pb}$ vs. (A) ${ }^{207} \mathrm{~Pb} / 204 \mathrm{~Pb}$ and (B) ${ }^{208} \mathrm{~Pb} /{ }^{004} \mathrm{~Pb}$. Data plotted are average compositions from Table 1. Leached residues are shown in detail in the insets in Figures 5A and B. Symbols are as in Figure 3, and fields as in Figure 2; data for NA-MORB field are from Dupré and Allègre (1980), Dupré et al. (1981), Hamelin et al. (1984), Sun (1980), and sources listed in Figure 2. The unradiogenic end of the NA-MORB field is defined by two samples (K) from the Kane Fracture Zone (Hamelin et al., 1984) and two samples from $17^{\circ} \mathrm{N}$ (Dosso et al., 1993); these are indicated separately from the main field.

again distinguished by their high ${ }^{208} \mathrm{~Pb} /{ }^{204} \mathrm{~Pb}$, indicating a high timeintegrated $\mathrm{Th} / \mathrm{Pb}$ ratio for the material leached. The gabbroic dikes from Site 920 are again the only exception in that the leachates have similar ${ }^{208} \mathrm{~Pb} /{ }^{204} \mathrm{~Pb}$ values to the leached residues. We do not wish to overinterpret the significance of the $\mathrm{Pb}$ leachate results because $\mathrm{Pb}$ concentrations have not been determined and thus blank corrections have not been made. Nevertheless, the consistency in both ${ }^{207} \mathrm{~Pb} /{ }^{204} \mathrm{~Pb}$ and ${ }^{208} \mathrm{~Pb} /{ }^{204} \mathrm{~Pb}$ would seem to us to be more than fortuitous.

tions of coexisting clinopyroxene and plagioclase. Leachates are shown as open circles and leached mineral residues as solid circles. Data plotted are average compositions from Table 1 . The $1 \sigma$ uncertainty for Nd measurements is shown on the figure; $1 \sigma$ error for $\mathrm{Sr}$ and $\mathrm{Pb}$ is within the size of the symbols. Diagonal lines represent equilibrium.

sition of the associated leached residues, whereas the leachates from all massif gabbros have high ${ }^{207} \mathrm{~Pb} /{ }^{204} \mathrm{~Pb}$. Furthermore, the leachates from Site 923 samples exhibit a very wide range of ${ }^{206} \mathrm{~Pb} / 204 \mathrm{~Pb}$ values as well, which extends to both lower and higher ${ }^{206} \mathrm{~Pb} / 204 \mathrm{~Pb}$ than seen in the leached residues.

$\mathrm{Pb}$-isotope systematics in ${ }^{208} \mathrm{~Pb} /{ }^{204} \mathrm{~Pb}$ vs. ${ }^{206} \mathrm{~Pb} /{ }^{204} \mathrm{~Pb}$ are similar to those in ${ }^{207} \mathrm{~Pb} /{ }^{204} \mathrm{~Pb}$, but it is interesting to note that the two leached mineral residues with relatively high $\Delta 7 / 4$ from Hole $923 \mathrm{~A}$ have similarly high ${ }^{208} \mathrm{~Pb} / 204 \mathrm{~Pb}$ for a given ${ }^{206} \mathrm{~Pb} /{ }^{204} \mathrm{~Pb}$ (Fig. $5 \mathrm{~B}$ ). Leachates are

\section{Oxygen Isotopes}

Results of O-isotope analyses are presented in Table 1. Mineral separates and whole-rock analyses of Leg 153 gabbros yield $\delta^{18} \mathrm{O}$ isotopic values between +5.5 and $+6.6 \%$ for most samples. In a plot of $\delta^{18} \mathrm{O}_{\text {plag }}$ vs. $\delta^{18} \mathrm{O}_{\text {cpx }}$ (Fig. 6), the data fall within the field of gabbro mineral separates from Hole $735 \mathrm{~B}$, but with a much smaller range of compositions. We observe that most Leg 153 gabbros plot within or near the regions of closed-system exchange. However, the data do not define a linear trend on this diagram as would be expected for closedsystem thermal reequilibration of a single bulk composition. Instead, the trend is nearly vertical, with plagioclase exhibiting a larger range of values $(+5.7$ to $+6.7 \%$ ) than clinopyroxene $(+5.3$ to $+5.8 \%)$. The 


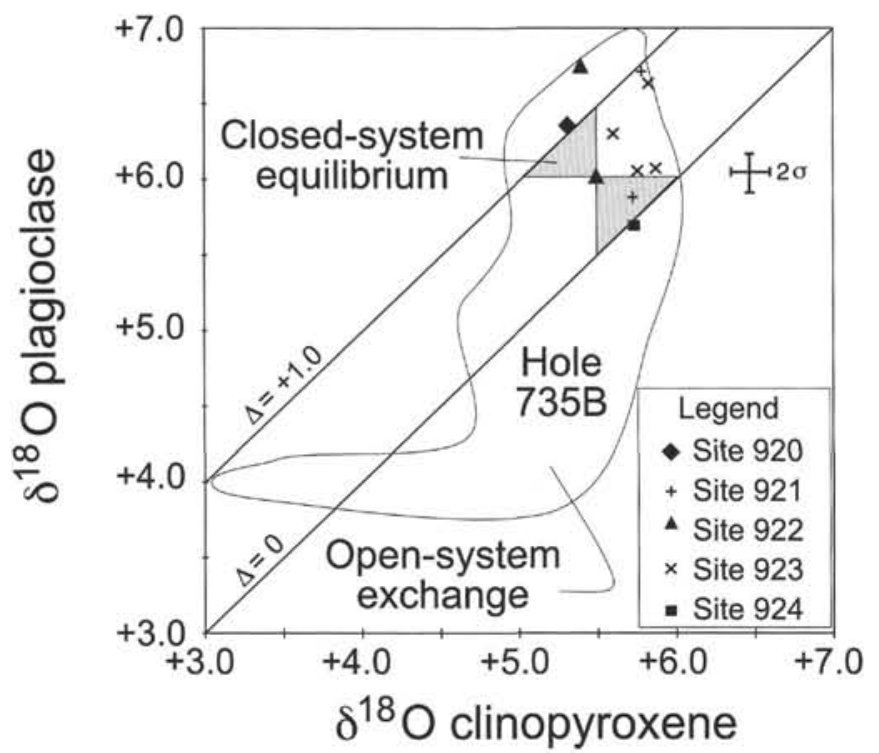

Figure 6. Plot of $\delta^{18} \mathrm{O}_{\text {cpx }}$ vs. $\delta^{18} \mathrm{O}_{\text {plag }}$ for gabbro samples from Leg 153. In this diagram, if the compositions equilibrated under closed-system conditions, coexisting minerals should exhibit small ${ }^{18} \mathrm{O}$ fractionation with small $\Delta_{x-y}=\delta^{18} O_{x}-\delta^{18} O_{y}$ values that form tight clusters or define linear arrays of $45^{\circ}$ slope that parallel the $\Delta=0$ fractionation line. At infinite temperature, $\Delta$ approaches zero, but with decreasing temperature, the array is displaced away from the $\Delta=0$ line. Plagioclase-clinopyroxene pairs quenched from basaltic magmas have $\Delta^{18} \mathrm{O}$ of $\sim 0.5 \%$. Assuming a $\delta^{18} \mathrm{O}$ value for average MORB of $+5.7 \%$, the corresponding primary magmatic $\delta^{18} \mathrm{O}_{\text {plag }}$ should be about $+6 \%$ and about $+5.5 \%$ for $\delta^{18} \mathrm{O}_{\text {cpx }}$. For closed-system equilibration to a different thermal regime, the trajectory of plagioclase-clinopyroxene pairs in $\delta^{18} \mathrm{O}$ vs. $\delta^{18} \mathrm{O}$ will have negative slopes and will fall within a right triangle similar to those shown. By contrast, open-system exchange with a new fluid composition results in curved mixing arrays that cut across the $\Delta=0$ fractionation line with steep positive slopes such as that shown by the field for gabbros from Hole 735B (Kempton et al., 1991). Symbols are as in Figure 3.

range of plagioclase values is even larger if Sample 153-920B-13R$4,56-65 \mathrm{~cm}$, is included $(+12 \%$ ). However, primary clinopyroxene is completely replaced by hornblende in this sample so the $\Delta$ value is unknown. This vertical trend reflects the fact that plagioclase more readily exchanges diffusively with a fluid to reflect the new $\delta^{18} \mathrm{O}$ value of a new hydrothermal condition. Clinopyroxene, in contrast, is more resistant to isotopic exchange with fluids and changes in $\delta^{18} \mathrm{O}$ are largely only through dissolution and reprecipitation of new phases such as amphibole.

In the basalt-seawater system, alteration at $250^{\circ}-300^{\circ} \mathrm{C}$ produces $\Delta_{\text {rock-water }}$ values of +5.0 to $+6.0 \%$. Hydrothermal alteration at higher temperatures $\left(>400^{\circ} \mathrm{C}\right)$ leads to lower $\Delta$ values and thus to depletion of the rock in ${ }^{18} \mathrm{O}$. Because the $\delta^{18} \mathrm{O}$ values for the Leg 153 gabbros range between +5.3 and $+6.7 \%$, we can conclude that they have undergone only limited high-temperature alteration. When the $\Delta_{\text {plag-cpx }}$ values are calculated for mineral pairs from the gabbros, the results indicate that they have crystallized from average MORB at or near the expected magmatic temperature (e.g., $\sim 1200^{\circ} \mathrm{C}$ for Samples 153 922B-3R-2, 39-44 cm, and 153-923A-16R-3, 9-15 cm, where $\Delta_{\text {plag- }}$ $c p x=0.5$ ). However, a few samples have $\delta^{18} \mathrm{O}_{\text {plag }}$ and $\delta^{18} \mathrm{O}_{\mathrm{cpx}}$ values that differ from the expected values for crystallization from average MORB, with $\Delta_{\text {plag-cpx }}$ trending away from 0.5 (Fig. 6). Samples with $\Delta_{\text {plag-cpx }}<0.5$ (e.g., Samples 153-921E-2R-2, 18-24 cm, 153-923A$10 \mathrm{R}-2,46-52 \mathrm{~cm}$, and $153-924 \mathrm{~B}-2 \mathrm{R}-1,16-20 \mathrm{~cm}$ ) may be attributed to limited exchange between the rock and seawater at high temperatures, whereas exchange reactions that have taken place at low temperatures can account for shifts in $\Delta_{\text {plag-cpx }}>0.5$ (e.g., Sample 153-
922A-2R-5, 69-76 cm). Prehnite replacement after plagioclase in Sample 153-920D-13R-2, 127-132 cm, has near magmatic values. A calculated temperature based on experimental $\Delta^{18} \mathrm{O}_{\text {prehnite-water }}\left(\mathrm{O}^{\prime} \mathrm{Neil}\right.$ and Taylor, 1967) yields a value of $\sim 250^{\circ} \mathrm{C}$.

\section{DISCUSSION}

The area of the Mid-Atlantic Ridge in the vicinity of the Kane Fracture Zone is characterized by lavas with relatively homogeneous major-element, trace-element and isotopic compositions (Dosso et al., 1993; Klein and Langmuir, 1987), and is recognized as a type example of N-MORB (i.e., normal MORB away from the influence of plumes). The new data presented here support this first-order observation, but further demonstrate that greater isotopic heterogeneity exists in the area than has previously been recognized. The scale of this heterogeneity cannot be fully constrained with the existing data set, but it is clear that geochemical variations exist both in time and space. In the following sections we will investigate the nature of this heterogeneity and its implications for mantle composition and magma chamber processes.

\section{Effects of Alteration}

Before we can assess and interpret the primary isotope systematics, it is necessary to identify those characteristics resulting from secondary alteration. As noted previously, samples for this study were selected to be as fresh as possible. Near magmatic O-isotope values confirm the relatively pristine nature of most samples analyzed (Table 1). However, not all plagioclase and clinopyroxene mineral pairs are in isotopic equilibrium (Fig. 6). Typical $\Delta_{\text {plag-cpx }}$ values for slowcooling plutonic rocks range between 0.8 and 1.3. Although many of the analyzed samples from Leg 153 are within this range, internal Oisotope fractionations between mineral phases $\left(\Delta_{\text {plag-cpx }}=0-1.3\right)$ suggest that the gabbros do not record a closed system and that some externally derived fluids are involved.

The Leg 153 gabbro data suggest that alteration took place, for the most part, at moderate to low temperatures $\left(200^{\circ}-300^{\circ} \mathrm{C}\right)$ where the fractionations between basalt and seawater are in the range of +5.0 to $+6.0 \%$. This contrasts markedly with gabbros from Hole $735 \mathrm{~B}$, where high-temperature interaction with seawater predominated (Kempton et al., 1991). The near-magmatic O-isotope values of most Leg 153 gabbros are also consistent with relatively low water/rock ratios $(<0.15)$ in most cases. However, such values are also recorded in gabbros that are as much as $70 \%$ altered (see Appendix), and low water/ rock ratios are clearly unrealistic for these rocks. Plagioclase from gneissic gabbro Sample 153-920D-13R-4, 56-65 cm, has a $\delta^{18} \mathrm{O}$ of $+12 \%$; this is indicative of significant low-temperature alteration and is consistent with the extensive replacement of the primary phases in this sample by chlorite and smectite. However, the amphibolized microgabbro (Sample 153-920B-13R-4, 30-38 cm) in contact with the gneissic gabbro (Sample 153-920D-13R-4, 56-65 cm), has a whole-rock $\delta^{18} \mathrm{O}$ of only $+5.6 \%$. This highlights the very localized nature of the alteration observed; indeed, this suggests that the gabbro must have served as a channel for low-temperature hydrothermal fluids, whereas the amphibolite was relatively impervious to them.

$\mathrm{Sr}$-isotope ratios also correlate with degree of alteration. For example, samples from Site 920 (total alteration $\sim 60 \%-75 \%$ ) have the highest $\mathrm{Sr}$-isotope ratios $(0.7027-0.7035)$, even after leaching. The acid leachates removed from these samples have even higher ${ }^{87} \mathrm{Sr} /{ }^{86} \mathrm{Sr}$ ratios (0.7045-0.7055; Table 1; Fig. 3). Similarly, clinopyroxenes from Site 922, where alteration is moderately high throughout the core $(\sim 10 \%-20 \%)$, have slightly elevated ${ }^{87} \mathrm{Sr} /{ }^{86} \mathrm{Sr}$ ratios $(0.7024$ 0.7025 ) compared to gabbros from Sites 921,923 , and 924 (average $\left.{ }^{87} \mathrm{Sr} /{ }^{86} \mathrm{Sr}=0.70236 \pm 2\right)$; again the leachates are even higher $(0.7040$ $0.7050)$. In fact, pyroxenes have consistently higher ${ }^{87} \mathrm{Sr} /{ }^{86} \mathrm{Sr}$ ratios 
than coexisting plagioclase, even after extensive leaching, and the leachates removed from clinopyroxenes are consistently higher than those removed from plagioclase (Fig. 4A). This is similar to the results from Hole 735B where gabbros show a large range in Sr-isotope composition, but where most of that variation is caused by alteration of the clinopyroxene, particularly in the upper part of Hole 735B where alteration and deformation are most intense.

These are not surprising observations, and can readily be explained by hydrothermal alteration and influx of seawater into the lower oceanic crust. Perhaps what is more surprising is that for rocks as altered as those from Site 920, the $\mathrm{Sr}$-isotope ratios are not higher than those measured. These results demonstrate two points. (1) Even where fluids have found ingress into the gabbroic crust, they have had limited compositional consequences, presumably because seawater was rapidly modified by interaction with the overlying basalt crust; the same observation was made by Kempton et al. (1991) for gabbros from Hole 735B. (2) Plagioclase may give a more accurate indication of the primary $\mathrm{Sr}$-isotope composition of the parental magma than clinopyroxene in most cases. Thus, the parental magmas for Leg 153 gabbros must have had a very limited range in Sr-isotope compositions (Figs. 3, 4), not only for gabbros within the same hole, but also for gabbros from different holes.

It has usually been assumed that $\mathrm{Pb}$ isotopes remain unaffected by hydrothermal alteration because the concentration of $\mathrm{Pb}$ in seawater is so low. Figure 7 demonstrates that most Leg 153 samples are consistent with this hypothesis. This figure summarizes the complex $\mathrm{Sr}$ $\mathrm{Pb}$ systematics for Leg 153 gabbros and leachates. In general, most leached residues show little variation in $\mathrm{Sr}$-isotope composition, with the exception of the extremely altered samples from Site 920 and the moderately altered samples from Site 922 (see inset to Fig. 7A). They also show only a small range in $\mathrm{Pb}$-isotope ratios for most samples, although on average gabbro minerals from Sites 920 and 922 have slightly higher ${ }^{206} \mathrm{~Pb} /{ }^{204} \mathrm{~Pb}$ (Fig. 7A) and those from Site 920 have higher ${ }^{208} \mathrm{~Pb} /{ }^{204} \mathrm{~Pb}$ (Fig. 7B) than most other gabbros from MARK.

When the ${ }^{206} \mathrm{~Pb} /{ }^{204} \mathrm{~Pb}$ vs. ${ }^{87} \mathrm{Sr} /{ }^{86} \mathrm{Sr}$ systematics of the leachates are considered, three trends are clearly defined. The predominant trend is toward strongly elevated ${ }^{87} \mathrm{Sr} /{ }^{86} \mathrm{Sr}$ values with only slight enrichment in ${ }^{206} \mathrm{~Pb}$ relative to the leached residues. A second trend exhibits less enrichment in ${ }^{87} \mathrm{Sr}$, but extends to higher ${ }^{206} \mathrm{~Pb} /{ }^{204} \mathrm{~Pb}$ values. The third trend shows slight to moderate enrichment in ${ }^{87} \mathrm{Sr}$, but unradiogenic $\mathrm{Pb}$-isotope values. Similar systematics are shown for ${ }^{208} \mathrm{~Pb} /{ }^{204} \mathrm{~Pb}$ vs. ${ }^{87} \mathrm{Sr} /{ }^{86} \mathrm{Sr}$ (Fig. 7B), but there is clearly a significant enrichment in ${ }^{208} \mathrm{~Pb}$ in the leachates from nearly all samples. Shown for reference in these figures are the compositions of serpentine separated from serpentinized harzburgite adjacent to websterite Sample 153-920D$15 \mathrm{R}-3,18-21 \mathrm{~cm}$, and secondary vein amphiboles from Site 920 (Kempton and Stephens, this volume), as well as pelagic sediment and Mn encrustations from the Mid-Atlantic-Ridge north of the Kane
Figure 7. Plot of ${ }^{87} \mathrm{Sr} /{ }^{86} \mathrm{Sr}$ vs. (A) ${ }^{206} \mathrm{~Pb} /{ }^{204} \mathrm{~Pb}$ and (B) ${ }^{208} \mathrm{~Pb} /$ ${ }^{204} \mathrm{~Pb}$ for gabbro mineral separates and leachates. Data plotted are average compositions from Table 1. Symbols for mineral separates and leachates as in Figure 3. Compositions of serpentine and secondary amphiboles are from Kempton and Stephens (this volume). Sediment field includes analyses of pelagic sediment and manganese encrustation on basalt from MARNOK (P.D. Kempton, unpubl. data). Curves are intended only to indicate trends and are not calculated mixing curves because fluid compositions are unknown. Data for leached residues are shown in an expanded view in the inset in $(A)$.
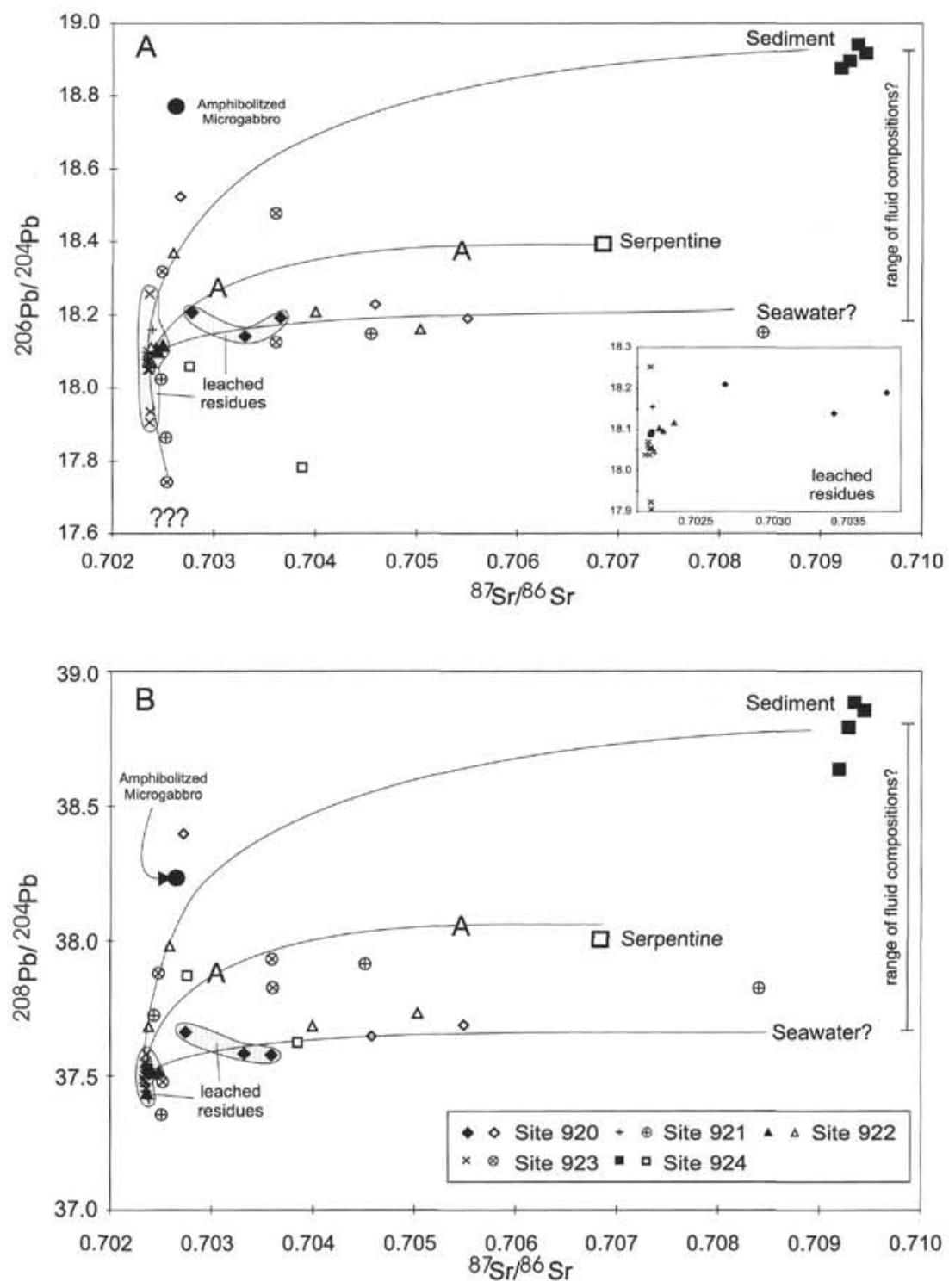
Fracture Zone (MARNOK). The value for seawater is hypothetical because, although the $\mathrm{Sr}$-isotope composition of seawater is well known, the $\mathrm{Pb}$-isotope composition is usually inferred from the compositions of sediments and Mn nodules. However, as recently demonstrated by Snow et al. (1993), there can be a significant component of continental crust in oceanic sediments for ${ }^{87} \mathrm{Sr}$, and the same is almost certainly true for $\mathrm{Pb}$. Thus, although seawater may dominate the composition of the hydrothermal fluids, those fluids may have different $\mathrm{Pb}$-isotope compositions locally, depending on the role of local sediments. Indeed, the trends on Figure 7 suggest that this is the case for the MARK area and that a range of fluid compositions may have been available, particularly with respect to $\mathrm{Pb}$ isotopes. The data indicate that for most samples, the hydrothermal fluids responsible for alteration had compositions indicative of a predominant seawater component. This may be because the $\mathrm{Pb}$ contents of the fluids were too low to effectively alter the gabbros, or because the isotopic compositions of the fluids were buffered to some average composition by the overlying basalt column, as suggested previously for $\mathrm{Sr}$ isotopes. Nevertheless, for some samples, it can be argued that sediments were also involved-one consequence being elevated ${ }^{206} \mathrm{~Pb} /{ }^{204} \mathrm{~Pb}$ and ${ }^{208} \mathrm{~Pb} /{ }^{204} \mathrm{~Pb}$.

However, the trend to low ${ }^{206} \mathrm{~Pb} /{ }^{204} \mathrm{~Pb}$ is enigmatic, particularly when the complex relationships between leachates and residual minerals from within some samples are considered. For example, in Sample 153-924B-2R-1, 16-20 cm, the leached residues of plagioclase and clinopyroxene have $\mathrm{Pb}$-isotope ratios (as well as ${ }^{87} \mathrm{Sr} /{ }^{86} \mathrm{Sr}$ and ${ }^{143} \mathrm{Nd} /{ }^{144} \mathrm{Nd}$ ) that are within error of each other; this would be expected if both minerals recently crystallized from the same magma. However, the leachate from the plagioclase, although having essentially the same ${ }^{206} \mathrm{~Pb} /{ }^{04} \mathrm{~Pb}$ as the plagioclase residue, has elevated ${ }^{207} \mathrm{~Pb} /$ ${ }^{204} \mathrm{~Pb}$ and ${ }^{208} \mathrm{~Pb} /{ }^{204} \mathrm{~Pb}$ (Table 1; Fig. 5). Similarly, the clinopyroxene leachate from the same rock has elevated ${ }^{207} \mathrm{~Pb} /{ }^{204} \mathrm{~Pb}$ and ${ }^{208} \mathrm{~Pb} /{ }^{204} \mathrm{~Pb}$, but much lower ${ }^{206} \mathrm{~Pb} / 204 \mathrm{~Pb}$. Thus, the leachable components of the different minerals are significantly different for $\mathrm{Pb}$ isotopes. One possible explanation is that the mineralogical constituents leached from the clinopyroxene (predominantly amphibole) differed from those leached from plagioclase (predominantly chlorite and/or smectite), and these secondary minerals probably formed at different temperatures, in the presence of different fluids. A similar situation occurs in Sample 153-921B-4R-2, 76-82 cm, where leachates from both clinopyroxene and plagioclase have higher ${ }^{207} \mathrm{~Pb} /{ }^{204} \mathrm{~Pb}$ than the residues, and the plagioclase-derived leachate has higher ${ }^{206} \mathrm{~Pb} /{ }^{204} \mathrm{~Pb}$ than the pyroxene-derived one.

However, whether a geological explanation for these variations is correct is difficult to determine with the information available. Although sediments from the area have higher ${ }^{207} \mathrm{~Pb} /{ }^{204} \mathrm{~Pb}$ and ${ }^{208} \mathrm{~Pb} /$ ${ }^{204} \mathrm{~Pb}$ than MORB and could easily have contributed to the compositions of the hydrothermal fluids, they also have more radiogenic ${ }^{206} \mathrm{~Pb} /{ }^{204} \mathrm{~Pb}$. To our knowledge there are no known compositions on the seafloor like those of the low ${ }^{206} \mathrm{~Pb} /{ }^{204} \mathrm{~Pb}$, high $\Delta 7 / 4, \Delta 8 / 4$, gabbro mineral leachates. Although we have not measured $\mathrm{Pb}$ contents in any of the mineral separates or leachates for this study, these are known to be very low in oceanic gabbros. Thus, even a small amount of contaminant can radically alter the measured $\mathrm{Pb}$-isotope composition. Kempton et al. (1993) found similar unradiogenic compositions for leachates from basalt glass dredged from MARNOK, and sug- gested that this component may result from contamination with anthropogenic $\mathrm{Pb}$. One possibility is that this contaminant was introduced during sample collection, handling, preparation, or analysis. We are currently investigating the latter two possibilities. However, we would emphasize that the two radiogenic leached residues from Sites 921 and 923 (Fig. 5) have been analyzed in duplicate and the replicate analyses agree well. Furthermore, Hamelin et al. (1984) report two unradiogenic $\mathrm{Pb}$ compositions for samples dredged from within the Kane Fracture Zone (Fig. 5), so there is evidence for the existence of a primary unradiogenic $\mathrm{Pb}$ component in the MARK area. In fact, these data confirm that some of the most unradiogenic $\mathrm{Pb}$ isotope compositions of the North Atlantic occur in the area of the Kane Fracture Zone. Only the two unusual samples from $17^{\circ} \mathrm{N}$ have lower values (Dosso et al., 1993).

\section{Spatial Variations and the Relationships Between Peridotites, Gabbroic Dikes, and Massif Gabbros}

One of the initial objectives of this study was to correlate, if possible, gabbro units from the different drill sites to place minimum constraints on the dimensions of magma chambers and/or the magmatic system(s) within the MARK area. Unfortunately, the relatively small range in isotopic compositions complicates this avenue of investigation. Excluding the amphibolitized microgabbro, gabbros from Sites 920 to 924 show amazing homogeneity in their average isotopic composition (Table 3 ). From this, we must conclude that $\sim 750,000$ yr ago either (1) the MARK area was a single magmatic system in which, if melts from multiple sources were injected, mixing was very efficient, or (2) the source was extremely homogeneous in isotopic composition over $\sim 20 \mathrm{~km}$ of ridge length such that the boundaries between different magmatic systems cannot be distinguished.

Although gabbros in the different holes are relatively homogeneous in their average isotopic compositions, there is nonetheless evidence for multiple source compositions beneath MARK. At its most extreme, the low $\mathrm{Nd}$ - and high $\mathrm{Pb}$-isotope ratios of the amphibolitized microgabbro and the high- $\mathrm{MgO}$ diabase dikes from Site 920 provide clear evidence of source heterogeneity (Figs. 2, 5). In total, the range of $\mathrm{Nd}$ - and $\mathrm{Pb}$-isotope compositions for magmatic rocks from MARK encompasses almost two-thirds of the compositional range exhibited by all of North Atlantic N-MORB (Figs. 2, 5). However, one might argue that these occurrences are rare and/or unrepresentative. Figure 8, however, demonstrates that isotopic heterogeneities are preserved even within the gabbro column, albeit more subtly. These figures show that, although there is no systematic change in isotopic composition with stratigraphic height, there is significant isotopic variation within a single hole. The consistency between the $\mathrm{Nd}$ - and $\mathrm{Pb}$-isotope results (Table 1) for individual minerals (i.e., those samples that show the greatest internal variation for $\mathrm{Pb}$ isotopes also show the greatest variation in ${ }^{143} \mathrm{Nd} /{ }^{144} \mathrm{Nd}$ ) suggests that these differences are not caused by alteration processes or by analytical uncertainty.

These within-sample variations will be discussed in detail in the following section. Here they provide evidence for the range of isotopic sources available beneath the MARK area $\sim 750,000 \mathrm{yr}$ ago. In particular, although the source was extremely homogeneous in its $\mathrm{Sr}$ -

Table 3. Average isotopic compositions of gabbros by site.

\begin{tabular}{llllll}
\hline & \multicolumn{1}{c}{ Site 920* } & \multicolumn{1}{c}{ Site 921 } & \multicolumn{1}{c}{ Site 922 } & \multicolumn{1}{c}{ Site 923 } & \multicolumn{1}{c}{ Site 924 } \\
\hline${ }^{87} \mathrm{Sr} /{ }^{86} \mathrm{Sr}$ & $0.70320 \pm 40$ & $0.70236 \pm 3$ & $0.70241 \pm 4$ & $0.70235 \pm 2$ & $0.70236 \pm 1$ \\
${ }^{143} \mathrm{Nd} / /^{144} \mathrm{Nd}$ & $0.513198 \pm 11$ & $0.513196 \pm 21$ & $0.513199 \pm 18$ & $0.513194 \pm 22$ & $0.513203 \pm 25$ \\
${ }^{206} \mathrm{~Pb} /{ }^{204} \mathrm{~Pb}$ & $18.186 \pm 44$ & $18.086 \pm 64$ & $18.079 \pm 44$ & $18.045 \pm 140$ & $18.090 \pm 21$ \\
${ }^{207} \mathrm{~Pb} / 204 \mathrm{~Pb}$ & $15.486 \pm 34$ & $15.456 \pm 18$ & $15.454 \pm 24$ & $15.487 \pm 39$ & $15.472 \pm 22$ \\
${ }^{200} \mathrm{~Pb} / 204 \mathrm{~Pb}$ & $37.612 \pm 74$ & $37.478 \pm 64$ & $37.481 \pm 60$ & $37.498 \pm 67$ & $37.512 \pm 41$ \\
$\delta^{18} \mathrm{O}$ (SMOW) & $+5.7 \pm 5$ & $+6.0 \pm 5$ & $+5.9 \pm 6$ & $+6.0 \pm 3$ & $+5.7 \pm 0$ \\
& & & & &
\end{tabular}

Notes: ${ }^{*}=$ averages exclude amphibolitized microgabbro, diabase dikes, leachates, and plagioclase with unusually high $\delta^{\mathrm{i}} \mathrm{O}$. If Sample $153-920 \mathrm{~B}-13 \mathrm{R}-4,56-65 \mathrm{~cm}$, is included, the average $\delta^{18} \mathrm{O}$ increases to $+8 \pm 4 \%$. 


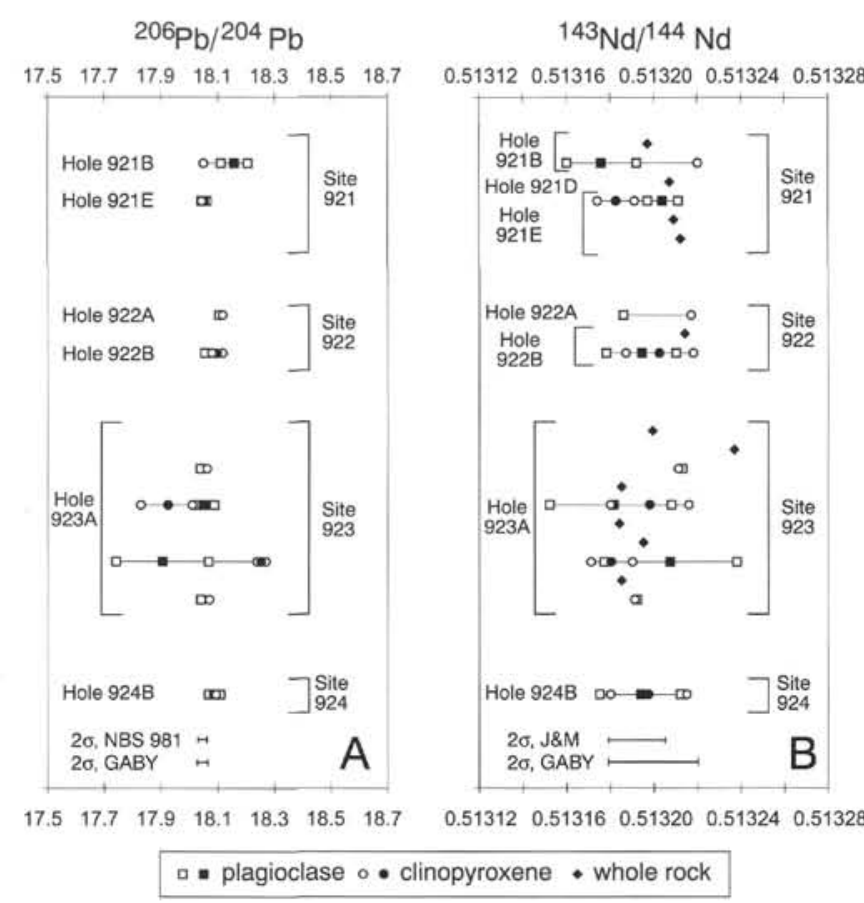

Figure 8. Diagram of (A) ${ }^{206} \mathrm{~Pb} /{ }^{204} \mathrm{~Pb}$ and (B) ${ }^{143} \mathrm{~N} /{ }^{144} \mathrm{Nd}$ values for individual (open squares and circles) and average (solid squares and circles) mineral analyses for massif gabbros from Sites 921, 922, 923, and 924. Also shown are unleached whole-rock ${ }^{143} \mathrm{Nd} /{ }^{144} \mathrm{Nd}$ data (solid diamonds) for massif gabbros from Agar et al. (this volume). The analyses are shown arranged by site. Multiple mineral analyses from the same sample are shown as overlapping symbols or connected by lines. Where more than one sample has been analyzed in a hole, they are arranged in stratigraphic order. Note that the vertical axis is only relative; it does not correspond to a depth measurement. The $2 \sigma$ reproducibility of machine standards NBS 981 and J\&M is indicated along with the $2 \sigma$ for GABY, the interlaboratory rock standard for Leg 153 .

isotope composition $\left({ }^{87} \mathrm{Sr} /{ }^{86} \mathrm{Sr}=0.70236 \pm 2\right)$, its $\mathrm{Nd}$ - and $\mathrm{Pb}$-isotope compositions were more variable, ranging from 0.51318 to 0.51322 in ${ }^{143} \mathrm{Nd} /{ }^{144} \mathrm{Nd}$ and from 17.9 to 18.3 in ${ }^{206} \mathrm{~Pb} /{ }^{204} \mathrm{~Pb}$. If the amphibolitized microgabbro is included the range increases to $0.51307-$ 0.51322 for ${ }^{143} \mathrm{Nd} / 144 \mathrm{Nd}$ and from 17.9 to 18.8 for ${ }^{206} \mathrm{~Pb} / 204 \mathrm{~Pb}$. If this larger range of compositions is commonly supplied to the magma system, to preserve the relative homogeneity exhibited by the gabbros (Table 3), it requires that (1) the input of the proportions of different magma types into the system remains fairly constant, and (2) mixing of the system must be very efficient, either occurring before entering the magma chamber or quickly afterward. Such a scenario is inconsistent with the low magma supply rate characteristic of the area today, and we have no reason to believe that the rate of magma supply was significantly greater in the past. We therefore consider it most likely that $\sim 1 \mathrm{Ma}$ the mantle source beneath MARK was relatively homogeneous and that compositions like the amphibolitized microgabbro are only infrequently put into the system. The small range of within-sample variations shown in Figure 8 may be typical of the range of compositions available to the magmatic system, but more data and further study are necessary to determine the relative frequency with which these variations or changes occur. An important implication of the relative isotopic homogeneity observed at MARK is that the trace-element composition of the source must be similarly homogeneous. Thus, most variations in trace-element concentrations and/ or ratios exhibited by the gabbros, and inferred for their parental magmas, must be the product of the melting regime and subsequent fractionation during magma chamber processes, and not variations in source composition.

Finally, although we can conclude that the gabbroic dikes from Site 920 must have been derived from a source with a similar compo- sition to that of the massif gabbros, we cannot unambiguously determine whether they were from the same magmatic system (i.e., whether they were cogenetic). Clearly, however, the peridotites from Site 920 are not directly the source of the Leg 153 gabbros (Figs. 2, 5). Even if the $\mathrm{Pb}$ and $\mathrm{Sr}$ differences can be explained by alteration and serpentinization, the higher Nd-isotope composition cannot be explained by alteration via seawater (which would leave the Nd-isotope composition unchanged) or by addition of a sediment component (which would lower the ${ }^{143} \mathrm{Nd} /{ }^{144} \mathrm{Nd}$ ratio).

\section{Within Sample Variations and Consequences for Magma Chamber Processes}

It has recently been observed that clinopyroxenes in oceanic gabbros from both Hole 735B and from the Mid-Cayman Rise have consistently higher $\mathrm{Nd}$-isotope compositions than coexisting plagioclase (Meen et al., 1993). Such variations in Nd-isotope compositions are enigmatic because the rare-earth elements are noted for their immobility during hydrothermal alteration of oceanic crust (Menzies and Seyfried, 1979). The young age of these rocks precludes an origin as a result of in situ radiogenic growth of ${ }^{143} \mathrm{Nd}$ after crystallization. Such variations are also not expected from simple models for cumulate formation, such as closed-system in situ crystallization or crystal-liquid sorting via density contrasts.

These isotopic variations support a model of complex magma chamber processes in which several scenarios could be envisaged: (1) coexisting cumulate phases may have crystallized from different magmas, (2) cumulus and intercumulus phases may crystallize from different magmas, (3) cumulus phases might be modified by interaction with migrating intercumulus liquid(s) of different compositions, either syn-or post-cumulate formation, or (4) some combination of the above. There is growing evidence to support this model. For example, Ross and Elthon (1993) have argued, on the basis of incompatible trace-element compositions, that mixing of compositionally distinct, small-degree fractional melts is recorded in some gabbros from Site 334 (Leg 37). In addition, observable isotopic disequilibrium exists between phenocrysts/xenocrysts and host basaltic glass for $\mathrm{Sr}$ and $\mathrm{Pb}$ for samples dredged from MARNOK (Kempton et al., 1993; P.D. Kempton, unpubl. data).

In this context, the $\mathrm{Nd}$-isotope data for Leg 153 gabbros are equivocal because the small range of values observed for the suite as a whole is only marginally greater than the external precision of the data (i.e., the analytical uncertainty of the data is best indicated by the external reproducibility of the J\&M standard solution, which is $\sim 50$ $\mathrm{ppm}, 2 \sigma$ ). In addition, clinopyroxene occurs with both higher and lower ${ }^{143} \mathrm{Nd} /{ }^{144} \mathrm{Nd}$ ratios than coexisting plagioclase (Figs. 4B, $8 \mathrm{~B}$ ). It was shown in Figure 2 that averages of multiple mineral analyses from the same sample lie within the $2 \sigma$ external reproducibility indicated by the J\&M Nd-isotope standard. Replicate analyses of the gabbroic rock standard (GABY) prepared as an interlaboratory standard during Leg 153 yield $0.513202 \pm 21(1 \sigma, \mathrm{n}=7)$, which is slightly greater than the variation on the J\&M standard solution. Nevertheless, Figure 8 shows that some isotopic variation exists outside of the range of external reproducibility for both $\mathrm{J} \& \mathrm{M}$ and GABY, particularly for $\mathrm{Pb}$ isotopes. This figure plots the ${ }^{206} \mathrm{~Pb} /{ }^{204} \mathrm{~Pb}$ and ${ }^{143} \mathrm{Nd} /{ }^{144} \mathrm{Nd}$ for individual mineral analyses for all massif gabbros analyzed in this study, along with the mineral averages where available and some unleached whole-rock ${ }^{143} \mathrm{Nd} /{ }^{144} \mathrm{Nd}$ data for massif gabbros from Agar et al. (this volume). The analyses are shown arranged by site. Where more than one sample is available in a hole, they are arranged in stratigraphic order. The differences between the average plagioclase and average clinopyroxene compositions of each sample agree within the external reproducibility of the data for most samples and in most cases, the replicate mineral analyses agree within $2 \sigma$ reproducibility of the GABY standard. Notable exceptions are Samples 153-921B4R-2, 76-82 cm, 153-923A-10R-2, 46-52 cm, and 153-923A-12R-1, $30-38 \mathrm{~cm}$. Furthermore, the differences between the maximum and minimum Nd-isotope values determined for any given sample is 
greater than the $2 \sigma$ external reproducibility for more than half of the samples analyzed. These results either mean that the analytical uncertainty is greater than that indicated by either the J\&M standard solution or the GABY rock standard, or that there is real variation in $\mathrm{Nd}$ isotopes outside of analytical uncertainty that has geologic significance. It may not be possible to distinguish between these two alternatives for the $\mathrm{Nd}$-isotope data, but it is interesting in this context that the variations in ${ }^{143} \mathrm{Nd} /{ }^{144} \mathrm{Nd}$ are mirrored in $\mathrm{Pb}$ isotopes (Figs. $4 \mathrm{C}$, $8 \mathrm{~A})$.

$\mathrm{Pb}$ isotopes are in equilibrium for most leached primary phases (i.e., coexisting plagioclase and clinopyroxene show good agreement in values) (Fig. 7A). However, for Samples 153-921B-4R-2, 76-82 $\mathrm{cm}, 153-923 \mathrm{~A}-10 \mathrm{R}-2,46-52 \mathrm{~cm}$, and $153-923 \mathrm{~A}-12 \mathrm{R}-1,30-38 \mathrm{~cm}$, there is clear disequilibrium in $\mathrm{Pb}$-isotope composition between coexisting plagioclase and clinopyroxene. Note that these differences are well outside of analytical uncertainty.

The results for Leg 153 gabbros are thus consistent with the models of Meen et al. (1993) and Ross and Elthon (1993), and demonstrate that melts derived from a range of source compositions (albeit a small one) have entered the sub-MARK magma chamber(s). These different isotopic signatures may arise by mixing of melts generated from isolated portions of depleted and enriched material within the mantle, or by melting that occurs at varying depths within a compositionally variable mantle. We cannot distinguish between these two models with our existing data set.

Preservation of within-sample isotopic heterogeneity like that seen in some Leg 153 gabbros may be sample specific. For example, in our sample set, the greatest heterogeneity is observed in poikilitic olivine gabbros. These relatively primitive lithologies are thought to be associated with the beginning of magmatic cycles (Shipboard Scientific Party, 1995b) where mixing between old and new magma batches would be expected. If the new magma batch has a different composition from that previously occupying the magma chamber, growth of oikocrystic olivine and clinopyroxene could enclose several generations of plagioclase of potentially different compositions, particularly if these magma chambers are largely crystal mushes rather than melt zones (Sinton and Detrick, 1992). Alternatively, latestage growth of intercumulus clinopyroxene from migrating melts having a different composition could similarly produce isotopic disequilibrium. More data from these lithologies are needed to distinguish among the various alternatives and to identify how frequently melts of different composition are input into the system.

\section{Mantle Dynamics and Source Variation with Time}

Considered collectively, gabbros, diabase dikes, and recent basalts from MARK exhibit a relatively large range in primary isotope compositions within a relatively small geographic area (Figs. 2, 5). This range is larger than previously recognized for this area (Dosso et al., 1993), and encompasses a significant proportion of the range of North Atlantic N-MORB. This is particularly significant when one considers that MARK is often accepted as a type locality for $\mathrm{N}$ MORB. Furthermore, the data presented here provide evidence for temporal changes in the isotopic composition of the mantle source in the MARK area over a period of $<1$ m.y.

The massif gabbros are estimated to be 0.75 m.y. in age. With few exceptions, they exhibit limited variations in $\mathrm{Sr}-, \mathrm{Nd}-$, and $\mathrm{Pb}$-isotope characteristics, and the mantle source inferred from them is similar from the middle of the spreading cell (Sites 921-923) to its boundary in the south (Site 920). However, recent basalts have significantly lower $\mathrm{Nd}$ - and higher $\mathrm{Pb}$-isotope compositions and there is almost no overlap between the two. Because the rocks are relatively young $(<0.75 \mathrm{Ma})$, these variations in isotopic signature cannot be the result of radioactive decay; nor can they be produced by secondary alteration processes for the reasons discussed previously. Therefore, to produce this variation, the rift valley basalts must have been from an isotopically distinct magmatic source compared with that for the older gabbros.
If we assume idealized seafloor spreading, with eruptions confined to the axis, and a spreading rate of $25 \mathrm{~mm} / \mathrm{yr}$, this corresponds to an overturn of $\sim 15 \mathrm{~km}$ of asthenosphere, and presumably a change in average mantle composition on that length scale. The change in compositions is apparently not smooth because Site 924, located approximately $2 \mathrm{~km}$ east of the other massif sites, and as much as 100,000 yr younger, shows no indication that the source composition has changed significantly in that time period. However, the peridotites at Site 920 may represent bits of upwelled asthenosphere now stranded in the lithosphere (Neumann and Forsyth, 1993) from an earlier stage in the evolution of the MARK area. If we include these rocks in our analysis, there is a clear, but gradual, shift in composition toward isotopically less depleted source with time, from among the most depleted compositions in the North Atlantic MORB to moderately enriched.

The high-MgO diabase dikes from Site 920 have compositions that have not been discovered among the spatially associated extrusive basalts, containing between 13 and $15 \mathrm{wt} \% \mathrm{MgO}$. This compares with the 5-7 wt\% MgO in dikes from Site 921 (Kempton and Casey, this volume) and in recent basalts from the neovolcanic zone (Reynolds, 1995). These unusual Mg-rich compositions are usually only observed as melt inclusions in phenocrysts. These magmas may represent one of the extreme end members predicted by the model discussed in this and the previous sections. The unusually enriched isotopic composition of the amphibolitized microgabbro may similarly represent one of the enriched components. The restriction of these unusual compositions to occurrence in the peridotite section, which is in turn located near to the accommodation zone, may not simply be fortuitous. Study of ridge segments to the north of the Kane Fracture Zone (MARNOK) have demonstrated that small volumes of unusual, enriched compositions are more likely to remain distinct and erupt at the edges of spreading cells where magma supply is lower. Toward the middle of spreading cells, enriched compositions become incorporated into existing magma chamber(s) and are homogenized.

\section{SUMMARY AND CONCLUSIONS}

1. There is no correlation between bulk composition and isotopic composition currently identified (i.e., oxide-rich gabbros having evolved compositions are not significantly different from more primitive olivine gabbros). There is also no systematic variation in primary isotopic composition either within a hole or between holes. Over the time scale of gabbro crystallization, as seen at Sites $921-924(\sim 100,000 \mathrm{yr})$, the same source or range of magmatic sources has been tapped. Small-scale variations outside of analytical uncertainty exist between mineral phases within a single sample, however, and these provide evidence for complex magma chamber processes, and periodic influx of melts with varying isotopic compositions.

2. O-isotope analyses of gabbro mineral separates and wholerock basalts and microgabbros have near primary magmatic values. $\delta^{18} \mathrm{O}_{\text {cpx }}$ vs. $\delta^{18} \mathrm{O}_{\text {plag }}$ systematics suggest relatively limited fluid-rock interaction, predominantly at moderate to low temperatures $\left(200^{\circ}-300^{\circ} \mathrm{C}\right)$. Combined $\mathrm{Sr}$ - and O-isotope evidence indicates that, even where fluids have found ingress into the gabbroic crust, they have had limited compositional consequences, presumably because seawater was rapidly modified by interaction with the overlying basalt crust. $\mathrm{Pb}$ isotopes suggest that, for most samples, the hydrothermal fluids responsible for alteration involved only seawater, but in a few cases it can be argued that sediments were also involved, resulting in slightly elevated $\mathrm{Pb}$-isotope ratios.

3. Two models can be proposed to explain the observable heterogeneity within the mantle at MARK. Different isotopic signatures are produced either by mixing between melts generated from isolated portions of depleted and enriched ma- 
terial within the mantle and/or are caused by melting at varying depths within a compositionally variable mantle.

4. We infer from the MARK gabbro data that the sub-MARK mantle is relatively homogeneous isotopically on a kilometer scale. This implies that its trace-element composition (as inferred from isotope ratios of $\mathrm{Sr}, \mathrm{Nd}$, and $\mathrm{Pb}$ ) must also be relatively homogeneous. Variations in trace-element concentrations and/or ratios of the gabbros and calculated parental magmas must, therefore, be the product of the melting regime and subsequent fractionation.

5. The average isotopic composition within the MARK area has changed over a period of $<1 \mathrm{~m}$.y., becoming more radiogenic in its $\mathrm{Pb}$-isotope composition but less radiogenic in its $\mathrm{Nd}$-isotope composition with time, indicating that the source is currently more enriched than that available $750,000 \mathrm{yr}$ earlier.

6. The most unusual enriched compositions (i.e., the amphibolitized microgabbro and high-MgO dikes) are found crosscutting the peridotite section, which occurs near the southern boundary of the spreading cell. Preservation of these distinctive compositions here may result from the lower magma supply rate expected at the boundaries of spreading cells.

\section{ACKNOWLEDGMENTS}

We would like to thank Jim Meen and an anonymous reviewer for their constructive comments on an earlier version of the manuscript. Samples of recent MARK basalts were kindly provided by J. Reynolds. Support for this work was provided by NERC grant GST/02/ 990. This paper represents NIGL Publication Series \# 159.

\section{REFERENCES}

Clayton, R.N., and Mayeda, T.K., 1963. The use of bromine pentafluoride in the extraction of oxygen from oxides and silicates for isotopic analysis. Geochim. Cosmochim. Acta, 27:43-52.

Cohen, R.S., Evensen, N.M., Hamilton, P.J., and O'Nions, R.K., 1980. U-Pb, $\mathrm{Sm}-\mathrm{Nd}$, and $\mathrm{Rb}-\mathrm{Sr}$ systematics of mid-ocean ridge basalt glasses. Nature, 283:149-153.

Cohen, R.S., and O'Nions, R.K., 1982. The lead, neodymium and strontium isotopic structure of ocean ridge basalts. J. Petrol., 23:299-324.

Craig, H., 1961. Standard for reporting concentrations of deuterium and oxygen-18 in natural waters. Science, 133:1833-1834.

Dick, H.J.B., Meyer, P.S., Bloomer, S., Kirby, S., Stakes, D., and Mawer, C., 1991. Lithostratigraphic evolution of an in-situ section of oceanic Layer 3. In Von Herzen, R.P., Robinson, P.T., et al., Proc. ODP, Sci. Results, 118: College Station, TX (Ocean Drilling Program), 439-538.

Dick, H.J.B., Natland, J., and Leg 147 Scientific Party, 1994. Melt transport and evolution in the shallow mantle beneath the East Pacific Rise: preliminary results from ODP Site 895. Mineral. Mag., 58A:229-230.

Dosso, L., Bougault, H., and Joron, J.L., 1993. Geochemical morphology of the North Atlantic Ridge, $10^{\circ}-24^{\circ} \mathrm{N}$ : trace element-isotope complementarity. Earth Planet. Sci. Lett., 120:443-462.

Dosso, L., Hanan, B.B., Bougault, H., Schilling, J.G., and Joron, J.L., 1991. $\mathrm{Sr}-\mathrm{Nd}-\mathrm{Pb}$ geochemical morphology between 10 degrees $\mathrm{N}$ and 17 degrees $\mathrm{N}$ on the Mid-Atlantic Ridge-a new MORB isotope signature. Earth Planet. Sci. Lett., 106:29-43.

Dungan, M.A., and Rhodes, J.M., 1978. Residual glasses and melt inclusions in basalts from DSDP Legs 45 and 46: evidence for magma mixing. Contrib. Mineral. Petrol., 67:417-431.

Dupré, B., and Allègre, C.J., 1980. Pb-Sr-Nd isotopic correlation and the chemistry of the North Atlantic mantle. Nature, 286:17-22.

Dupré, B., Lambret, B., Rousseau, D., and Allègre, C.J., 1981. Limitations of the scale of mantle heterogeneities under ocean ridges. Nature, 294:552554.

Frey, F.A., Walker, N., Stakes, D., Hart, S.R., and Nielsen, R., 1993. Geochemical characteristics of basaltic glasses from the AMAR and FAMOUS axial valleys, Mid-Atlantic Ridge $\left(36^{\circ}-37^{\circ} \mathrm{N}\right)$ : petrogenetic implications. Earth Planet. Sci. Lett., 115:117-136.

Hamelin, B., Dupré, B., and Allègre, C.J., 1984. Lead-strontium isotopic variations along the East Pacific Rise and the Mid-Atlantic Ridge: a comparative study. Earth Planet. Sci. Lett., 67:340-350.
Humler, E., and Whitechurch, H., 1988. Petrology of basalts from the Central Indian Ridge (lat. $25^{\circ} 23^{\prime} \mathrm{S}$, long. $70^{\circ} 04 \mathrm{E}$ ): estimates of frequencies and fractional volumes of magma injection in a two layered reservoir. Earth Planet. Sci. Lett., 88:169-181.

Ito, E., White, W.M., and Göpel, C., 1987. The O, Sr, Nd and Pb isotope geochemistry of MORB. Chem. Geol., 62:157-176.

Johnson, K.T.M., and Dick, H.J.B., 1992. Open system melting and temporal and spatial variation of peridotite and basalt at the Atlantis II fracture zone. J. Geophys. Res., 97:9219-9241.

Johnson, K.T.M., Dick, H.J.B., and Shimizu, N., 1990. Melting in the oceanic upper mantle: an ion microprobe study of diopsides in abyssal peridotites. J. Geophys. Res., 95:2661-2678.

Karson, J.A., 1991. Accommodation zones and transfer faults: integral components of Mid-Atlantic Ridge extensional systems. In Peters, U.J., Nicolas, A., and Coleman, R.G. (Eds.), Ophiolites Genesis and Evolution of Oceanic Lithosphere: Dordrecht (Kluwer Academic), 21-37.

Kempton, P.D., Hawkesworth, C.J., and Fowler, M., 1991. Geochemistry and isotopic composition of gabbros from layer 3 of the Indian Ocean crust, Hole 735B. In Von Herzen, R.P., Robinson, P.T., et al., Proc. ODP, Sci. Results, 118: College Station, TX (Ocean Drilling Program), 127-143.

Kempton, P.D., Lawson, N.K., Pearce, J.A., and Browning, P., 1993. Isotopic and chemical variations along the Mid-Atlantic Ridge north of the Kane Fracture Zone [paper presented at IAVCEI, Canberra, Australia]: Ancient Volcanism and Modern Analogues, 57. (Abstract)

Klein, E.M., and Langmuir, C.H., 1987. Global correlations of ocean ridge basalt chemistry with axial depth and crustal thickness. J. Geophys. Res. 92:8089-8115.

Machado, N., Ludden, J.N., Brooks, C., and Thompson, G., 1982. Fine scale isotopic heterogeneity in the sub-Atlantic mantle. Nature, 295:226-229.

Meen, J.K, Elthon, D., Ross, D.K., and Collins, D.T., 1993. Open-system magmatic processes indicated by trace element and isotopic chemistry of Mid-Cayman Rise gabbros. Eos, 74:653. (Abstract)

Menzies, M., and Seyfried, W.E., 1979. Basalt-seawater interaction: trace element and strontium isotopic variations in experimentally altered glassy basalt. Earth Planet. Sci. Lett., 44:463-472.

Neumann, G.A., and Forsyth, D.W., 1993. The paradox of the axial profile: isostatic compensation along axis of the Mid-Atlantic Ridge. J. Geophys. Res., 98:17891-17910.

O'Neil, J.R., and Taylor, H.P., Jr., 1967. The oxygen isotope and cation exchange chemistry of feldspars. Am. Mineral., 52:1414-1437.

Purdy, G.M., and Detrick, R.S., 1986. Crustal structure of the Mid-Atlantic Ridge at $23^{\circ} \mathrm{N}$ from seismic refraction studies. J. Geophys. Res., 91:3739-3762.

Reynolds, J.R., 1995. Segment-scale systematics of mid-ocean ridge magmatism and geochemistry [Ph.D. dissert.]. Columbia Univ., Palisades, NY.

Richards, P., Shimizu, P., and Allègre, C.J., 1976. ${ }^{143} \mathrm{Nd} /{ }^{143} \mathrm{Nd}$, a natural tracer: an application to oceanic basalt. Earth Planet. Sci. Lett., 31:269278.

Ross, K., and Elthon, D., 1993. Cumulates from strongly depleted midocean-ridge basalt. Nature, 365:826-829.

Shipboard Scientific Party, 1995a. Site 920. In Cannat, M., Karson, J.A., Miller, D.J., et al., Proc. ODP, Init. Repts., 153: College Station, TX (Ocean Drilling Program), 45-119.

1995b. Site 923. In Cannat, M., Karson, J.A., Miller, D.J., et., Proc. ODP, Init. Repts., 153: College Station, TX (Ocean Drilling Program), 217-258.

Sinton, J.M., and Detrick, R.S., 1992. Mid-ocean ridge magma chambers. J. Geophys. Res., 97:197-216.

Snow, J., 1993. The isotope geochemistry of abyssal peridotites and related rocks. [Ph.D. dissert.]. Massachusetts Institute of Technology/Woods Hole Oceanographic Institution Joint Program in Oceanography.

Sobolev, A.V., and Shimizu, N., 1993. Ultra-depleted primary melt included in an olivine from the Mid-Atlantic Ridge. Nature, 363:151-154.

Sun, S.-S., 1980. Lead isotopic study of young volcanic rocks from midocean ridges, ocean islands and island arcs. Philos. Trans. R. Soc. London A, 297:409-445.

Todt, W., Cliff, R.A., Hanser, A., and Hofmann, A.W., $1984 .{ }^{202} \mathrm{~Pb}$ and ${ }^{205} \mathrm{~Pb}$ double spike for lead isotopic analyses. Terra Cognita, 4:209. (Abstract)

White, W.M., and Hofmann, A.W., 1982. Sr and Nd isotope geochemistry of oceanic basalts and mantle evolution. Nature, 296:821-825.

\footnotetext{
Date of initial receipt: 3 August 1995

Date of acceptance: 14 December 1995

Ms 153SR-020
} 


\section{APPENDIX}

\section{Petrographic Descriptions}

Sample 153-920B-13R-4, 30-36 cm: Amphibolitized microgabbro. Fine grained. Sample consists of $50 \%$ plagioclase, $26 \%$ clinopyroxene, $2 \%$ iron oxide minerals, and $22 \%$ brown amphibole. Texture is mosaic equigranular; deformation twins are present in some plagioclase. Clinopyroxene porphryoclasts are strained and recrystallized to strain-free neoblasts; plagioclase is totally recrystallized. Brown amphibole replaces clinopyroxene, and is associated with iron oxide minerals. Foliation is defined by alternating bands rich in plagioclase or rich in clinopyroxene + amphibole. Minor chlorite is present in thin crosscutting veinlets, but most of sample is unaffected by lowtemperature alteration $(<2 \%)$.

Sample 153-920B-13R-4, 56-65 cm: Gneissic oxide gabbro. Coarse grained. Original mineral proportions are difficult to estimate because of extensive alteration, but $\sim 75 \%$ plagioclase, $20 \%$ pyroxene, and $5 \%$ iron oxide minerals; orthopyroxene may be present as well as clinopyroxene. Pyroxenes are partially recrystallized and are surrounded by neoblasts; most pyroxene is replaced by brown amphibole. Plagioclase is extensively recrystallized into neoblasts $0.5-0.75 \mathrm{~mm}$ in size. Large strained porphyroclasts have deformation twins and are partially replaced by chlorite; neoblasts are less altered than primocrysts. Oxides form the main interstitial phase; they concentrate in bands where they partially to completely enclose pyroxene neoblasts. Total alteration is $\sim 60 \%$. Large masses of chlorite \pm smectite replace both pyroxene and plagioclase. The rock contains numerous subhedral/euhdral crystals with low birefringence and moderate relief.

Sample 153-920D-13R-2, 127-132 cm: Pegmatitic oxide gabbro. The sample is composed of $45 \%$ pyroxene, with $48 \%$ prehnite + chlorite + epidote after plagioclase and $7 \%$ iron oxide minerals. Orthopyroxene may be present as well as clinopyroxene. Note that the shipboard description of this particular gabbroic vein reports significantly more clinopyroxene $(85 \%)$ and less plagioclase $(10 \%)$. No original plagioclase is preserved, but it appears to have been partially recrystallized prior to replacement. Clinopyroxene is largely replaced by brown and green amphibole, but the cores of larger grains are relatively free of alteration. Clinopyroxene is partially recrystallized; locally bands of neoblasts cut across large porphyroclasts, but most neoblasts form bands along with stringers of iron oxide minerals, defining an incipient foliation. Calcite occurs in trace amounts in vugs. Total alteration is $~ 70 \%$.

Sample 153-921B-4R-2, 76-82 cm: Poikilitic olivine gabbro. Medium grained. Sample consists of $20 \%$ olivine, $70 \%$ plagioclase, $10 \%$ green clinopyroxene. Olivine forms anhedral subophitic grains that partially enclose cumulus plagioclase. It is partially replaced by chlorite, smectite, and magnetite. Clinopyroxene forms poikilitic grains with only minor alteration. Plagioclase is the main cumulus phase, grains range from 2 to $40 \mathrm{~mm}$ in size; most are unaltered, with only minor sericite along cracks. Total alteration is $<5 \%$. Oxides are limited to magnetite after olivine.

Sample 153-921E-2R-2, 18-24 cm: Pegmatitic gabbro. The sample consists of $45 \%$ brown clinopyroxene, $45 \%$ plagioclase, $10 \%$ olivine, and $<1 \%$ iron oxide minerals. Total alteration is $1 \%-2 \%$. Olivine occurs as subhedral crystals $0.5-2.5 \mathrm{~mm}$, only slightly replaced by magnetite + smectite \pm chlorite \pm amphibole \pm talc. Clinopyroxene forms grains up to $25 \mathrm{~mm}$ in size. These are typically subhedral, and some display fine-scale exsolution. Most are unaltered, but all rims are partially replaced by brown and green amphibole \pm magnetite. Plagioclase forms anhedral grains $>0.5 \mathrm{~mm}$ to several centimeters in size. These are largely unaltered. Opaque minerals are anhedral and interstitial, $<<1 \%$.

Sample 153-922A-2R-5, 69-76 cm: Oxide gabbro. The sample consists of $15 \%$ brown clinopyroxene, $3 \%$ orthopyroxene, $75 \%$ plagioclase, and $7 \%$ iron oxide minerals. The sample is extensively deformed. Clinopyroxene forms subhedral pinkish brown grains, with porphyroclasts up to $6 \mathrm{~mm}$ in size; it is partially replaced by chlorite \pm magnetite \pm actinolite \pm smectite along cracks, cleavage planes, and along the rims. Minor exsolution of orthopyroxene is present. Orthopyroxene (up to $2 \mathrm{~mm}$ ) forms anhedral grains with moderate to strong alteration along rims and cracks, and is replaced by talc \pm magnetite \pm smectite. Plagioclase is strongly recrystallized; original crystals were up to $1 \mathrm{~cm}$ in size, but neoblasts are $<1.5 \mathrm{~mm}$. Unrecrystallized porphyroclasts are strained and show deformation twins. Iron oxide minerals form distinct bands that crosscut the section. They appear to flow around the primary mineral phases, partially to completely enveloping plagioclase neoblasts, ranging to interstitial between neoblasts. Total alteration is $\sim 20 \%$. Olivine occurs at one end of the thin section, which is partially replaced by talc + magnetite. This olivine is probably related to the adjacent olivine gabbro unit and not to the oxide gabbro.

Sample 153-922B-3R-2, 39-44 cm: Poikilitic olivine gabbro.The sample consists of $10 \%$ olivine, $40 \%$ green clinopyroxene, and $50 \%$ plagioclase.
Most of the sample is one large, optically continuous clinopyroxene enclosing subhedral cumulus plagioclase. Clinopyroxene has green cores and is zoned to brown toward the rims; it is replaced by brown amphibole on the rims and along cleavage planes. Olivine forms anhedral grains largely replaced by talc and amphibole (tremolite?), smectite, and magnetite; grain size ranges up to $4 \mathrm{~mm}$. Plagioclase is $<10 \mathrm{~mm}$ in size. It is pristine with no obvious alteration, but is crosscut by fine veinlets of chlorite. Total alteration is $\sim 10 \%$.

Sample 153-923A-6R-1, 26-31 cm: Olivine gabbro. Medium grained. The sample exhibits grain-size layering and a preferred crystal orientation defined by preferentially aligned, elongate mafic phases. The sample consists of $5 \%$ olivine, $55 \%$ plagioclase, $40 \%$ brown clinopyroxene, and $<<1 \%$ iron oxide minerals. Olivine shows minor alteration to talc + magnetite; minor replacement of clinopyroxene by brown amphibole along grain boundaries and cleavage planes, plus minor alteration to actinolite \pm chlorite. Clinopyroxene has fine-scale exsolution of orthopyroxene, and exhibits complex intergrowth textures between adjacent pyroxenes with different crystallographic orientations. Total alteration is $<1 \%$.

Sample 153-923A-10R-2, 46-52 cm: Olivine gabbro. This sample occurs near the top of magmatic cycle \#6 as defined during shipboard analysis (Shipboard Scientific Party, 1995b). Medium grained. The sample consists of $20 \%$ olivine, $30 \%$ clinopyroxene, and $50 \%$ plagioclase. The sample exhibits a preferred crystal orientation defined by preferentially aligned, elongate, mafic phases. Olivine forms anhedral cumulate crystals $\sim 5 \mathrm{~mm}$ in size. It is partially altered to talc + magnetite along cracks; it shows minor alteration to chlorite. Clinopyroxene is anhedral and $\sim 5 \mathrm{~mm}$ in size. It exhibits complex intergrowth textures between adjacent pyroxenes with different crystallographic orientations. Aside from minor replacement by brown amphibole along rims and in areas of complex crystal intergrowths, clinopyroxene is unaltered. Plagioclase is anhedral and locally shows deformation lamellae and recrystallization into neoblasts. Oxide occurrence is confined to magnetite after olivine alteration. Total alteration is $1 \%-2 \%$.

Sample 153-923A-12R-1, 30-38 cm: Poikilitic olivine gabbro. Medium to coarse grained. The sample consists of $35 \%$ olivine, $45 \%$ green clinopyroxene, and $20 \%$ plagioclase. This sample forms the companion to Sample 153 $923 \mathrm{~A}-10 \mathrm{R}-2,46-52 \mathrm{~cm}$, and is near the bottom of magmatic cycle \#6. Olivine exhibits classic crescumulate texture and is largely unaltered, but has minor alteration to talc and magnetite and replacement by chlorite and/or smectite along fine cracks. A diffuse vein of tremolite + talc occurs at one end of the sample; adjacent to the vein, olivine is more extensively replaced by these phases. Clinopyroxene has a dusty appearance resulting from numerous minute inclusions. It occurs as large poikilitic crystal $60 \mathrm{~mm}$ in length. There is some secondary alteration along cleavage planes. Green cores zone to pale brown clinopyroxene at distal edges of poikilitic crystals. Plagioclase is anhedral to subhedral where included in clinopyroxene or olivine; it ranges from 2 to $10 \mathrm{~mm}$ in size. Larger grains exhibit alteration to sericite \pm chlorite along thin veinlets, especially where near altered olivine. Magnetite occurs after olivine along cracks and in trace amounts as a primary interstitial phase. Trace of pale brown amphibole occurs interstitially, especially adjacent to oxides and along some olivine + plagioclase grain boundaries; it is probably magmatic. Total alteration is $5 \%-10 \%$

Sample 153-923A-16R-3, 9-15 cm: Poikilitic olivine gabbro. Medium grained. The sample consists of $22 \%$ olivine, $18 \%$ green clinopyroxene, $60 \%$ plagioclase, and $<1 \%$ iron oxide minerals. Oikocrystic olivine encloses small euhedral plagioclase; poikilitic clinopyroxene partially encloses larger subhedral plagioclase. Olivine appears to be concentrated into a single layer. Plagioclase and clinopyroxene are largely unaltered. Oxides are limited to magnetite after olivine. Olivine is altered to talc, magnetite, chlorite/smectite. Trace of brown amphibole after clinopyroxene and olivine is observed, especially along grain boundaries.

Sample 153-924B-2R-1, 16-20 cm: Olivine gabbro. Medium to coarse grained. The sample consists of $5 \%$ olivine, $55 \%$ clinopyroxene, and $40 \%$ plagioclase. Subhedral olivine is a cumulate phase largely altered to smectite, talc, and magnetite, particularly along cracks. Grains are typically $2-3 \mathrm{~mm}$ in size. Clinopyroxene ranges from skeletal green oikocrysts that enclose euhedral cumulus plagioclase laths to less common brown cumulus(?) crystals that do not contain plagioclase; it contains fine-scale exsolution lamellae of orthopyroxene (?). Clinopyroxenes exhibit complex intergrowth textures along grain boundaries, and other pyroxenes have a different crystallographic orientation. Minor alteration occurs where the mineral is adjacent to olivine. Subhedral plagioclase is a cumulus phase. Alteration to chlorite \pm sericite is present along microcracks, but otherwise the mineral is largely unaltered. Thin chlorite veinlets crosscut the section, altering all phases. Total alteration is $<2 \%-3 \%$. 